\title{
The Heavy Quark Potential from Wilson's Exact Renormalization Group
}

\author{
Ulrich ELLWANGER \\ Laboratoire de Physique Théorique et Hautes Energies[? \\ Université de Paris XI, bâtiment 211, 91405 Orsay Cedex, France \\ Manfred HIRSCH$]^{3}$ and Axel WEBER' \\ Institut für Theoretische Physik \\ Universität Heidelberg, Philosophenweg 16, D-69120 Heidelberg, FRG \\ and \\ Laboratoire de Physique Théorique et Hautes Energies ${ }^{2}$ \\ Université de Paris XI, bâtiment 211, 91405 Orsay Cedex, France
}

\begin{abstract}
We perform a calculation of the full momentum dependence of the gluon and ghost propagators in pure SU(3) Yang-Mills theory by integrating Wilson's exact renormalization group equations with respect to an infrared cutoff $k$. The heavy quark potential in the quenched approximation can be expressed in terms of these propagators. Our results strongly indicate a $1 / p^{4}$ behaviour of the heavy quark potential for $p^{2} \rightarrow 0$. We show in general, that effective actions which satisfy Schwinger-Dyson equations, correspond to (quasi-) fixed points of Wilson's exact renormalization group equations.
\end{abstract}

LPTHE Orsay 96-50, HD-THEP-96-21, ICN-UNAM-96-08

\footnotetext{
${ }^{1} \mathrm{e}-$ mail: ellwange@qcd.th.u-psud.fr

${ }^{2}$ Laboratoire associé au Centre National de la Recherche Scientifique - URA D0063

${ }^{3}$ Supported by a DAAD-fellowship HSP II financed by the German Federal Ministry for Research and Technology; e-mail: m.hirsch@thphys.uni-heidelberg.de

${ }^{4}$ Supported by a LGFG-fellowship of the Land Baden-Württemberg and a DAADfellowship; current address: Instituto de Ciencias Nucleares, Universidad Nacional Autónoma de México, Circuito Exterior, A.P. 70-543, 04510 México, D.F., Mexico; e-mail: axel@nuclecu.unam.mx
} 


\section{Introduction}

The computation of reliable phenomenological numbers on the basis of non-perturbative QCD is still an unsolved problem. Numerical methods seem to be required, as the simulation of the theory on a space-time lattice. A semi-phenomenological tool is provided by the Schwinger-Dyson equations (SDEs), which are reviewed in this respect in [1]. In the present paper we discuss an approach to non-perturbative phenomena in non-abelian gauge theories, which is based on the integration of exact renormalization group equations (ERGEs) [2] in continuum quantum field theory [3].

ERGEs describe the continuous evolution of effective Lagrangians, or effective actions, with a scale (or infrared cutoff) $k$, and allow to obtain the full quantum effective action for $k \rightarrow 0$ from a bare (microscopic) effective action at a cutoff scale $k=\Lambda$. In contrast to standard renormalization group equations they describe this evolution including all irrelevant couplings, or higher dimensional operators, and are exact in spite of the appearance of only one-loop diagrams. The price to pay is the fact that one has to deal with an infinite system of coupled differential equations which for practical purposes requires some truncation. The types of approximations or systematic expansions, which can be employed, depend on the phenomena under consideration:

One can expand the effective Lagrangian (or Hamiltonian or action) in powers of momenta or derivatives, but keep all powers of the involved field. This kind of expansion within the ERGEs is appropriate for the calculation of effective potentials, which are required to study the existence and nature of phase transitions (see [4] for some early literature).

Alternatively, one can expand the effective action in powers of fields, keeping all powers of the momenta [5]. This allows, e.g., to study the formation of bound states by using ERGEs to look for the appearance of poles in four-point functions [6]. Finally, in order to describe dynamical symmetry breaking, composite fields can be introduced [7, 8] and different expansions can be applied to the parts of the effective action involving fundamental and composite fields, respectively.

The ansatz of the present paper is based on the expansion of the effective action in powers of fields, which allows to study the full momentum dependence of the (gluon and ghost) propagators. Within this expansion the knowledge of the running 3 - and 4-point functions is required in order to 
integrate the ERGE for the running of the 2-point function, the knowledge of the 4- and 5-point functions is required for the ERGE of the 3-point function etc. $[5,6]$.

In the case of gauge theories this expansion gets to some extent reorganized: due to gauge invariance (or, more precisely, BRST invariance) parts of higher $\mathrm{N}$-point functions (with certain Lorentz and gauge index structures) are completely determined by lower $\mathrm{N}$-point functions. The usual procedure consists in imposing Slavnov-Taylor identities (STIs) on the effective action, which can be solved for "dependent" parts of $\mathrm{N}$-point functions in terms of "independent" ones. For instance, in the present case of a pure Yang-Mills theory a gauge invariant term in the effective action of the form

$$
F_{\mu \nu}^{a}\left(f\left(D^{2}\right) F_{\mu \nu}\right)^{a}
$$

(where $D$ denotes the covariant derivative and $f$ an arbitrary function) describes simultaneously the "independent" effective gluon propagator or 2point function, and certain "dependent" Lorentz and gauge index structures of the 3-, 4- and higher gluonic N-point functions. Additional "independent" structures of the gluonic 3- and higher $\mathrm{N}$-point functions are described in terms of gauge invariant expressions involving at least three powers of the field strength $F_{\mu \nu}$.

The application of ERGEs to gauge theories faces some technical problems, since ERGEs are based on an intermediate infrared momentum cutoff $k$, and such a cutoff generally breaks gauge or BRST invariance [9-14]. One way to deal with these problems is the modification of the STIs $[11,13,14]$ : the modified STIs impose "fine tuning conditions" on those couplings in the effective action at $k \neq 0$, which break gauge or BRST invariance. These "fine tuning conditions" are such that they guarantee the BRST invariance of the effective action for $k \rightarrow 0$.

As a consequence of the (modified) STIs, at any value of $k$, the "dependent" parts of the effective action can thus be obtained either via the modified STIs in terms of the "independent" parts at the scale $k$, or directly from the integration of the ERGEs. Below we will employ approximations which consist in neglecting contributions to the r.h.sides of the ERGEs. In the absence of approximations, both methods to obtain the dependent parts lead to identical results. In the presence of approximations one should determine the dependent parts of the effective action from the modified STIs. 
Approximations on the r.h.sides of ERGEs for independent parts of the effective action do thus not imply a violation of the modified STIs.

In the presence of approximations one can nevertheless study the ERGEs for the dependent parts of the effective action and see, whether these ERGEs deviate strongly from the corresponding equations obtained from the $k^{-}$ derivatives of the modified STIs. As emphasized in [14], this method allows for a numerical self-consistency check of the approximation and will also be employed in the present paper.

As described in section 3, our approximation consists in the neglect of the contribution of certain parts of the 3- and 4-point functions to the ERGEs of the 2-point functions. We take those parts of the 3 - and 4 -point functions into account, however, which are determined in terms of the 2-point functions through the standard part of the STIs. In the purely gluonic sector these are those obtained by an expansion of eq. (1.1) in powers of the gauge field $A_{\mu}^{a}$.

No approximation is imposed on the momentum dependence of the 2 point functions (the gluon and ghost propagators). These propagators by themselves are gauge dependent and not directly observable quantities. A certain combination thereof, however, has a physical meaning: The potential between infinitely heavy quarks can be written as a product of the dressed quark-gluon vertices and the full gluon propagator, and the quark-gluon vertex function is given by the ghost propagator function. As a result the heavy quark potential depends on both propagator functions, which we calculate here in the quenched approximation.

The aim of this paper is to see, how much information on the heavy quark potential in quenched QCD can be obtained within the approximation we employ, which is seen to be self-consistent for a range of momenta $p^{2}$ down to a certain value $p^{2} \sim k_{0}{ }^{2}$ (see section 5 ). We find strong evidence for a $1 / p^{4}$-behaviour within the trustworthy range of $p^{2}$.

Both the method and the results can be compared with the SchwingerDyson formalism. To this end we derive in section 2 a new and general relation between ERGEs and SDEs: we show that effective actions, which satisfy SDEs in the presence of an infrared cutoff $k$, are (quasi-) fixed points of the ERGEs. Thus a search for fixed points of the ERGEs for $k \rightarrow 0$ is equivalent to the search for solutions of SDEs.

Investigations of the gluon propagator have been performed in the context of SDEs both in the axial [15, 16] and Landau [17] gauge. In most cases solutions with a singular behaviour like $1 / p^{4}$ have been found to be 
consistent. (A behaviour less singular than $1 / p^{2}$ has been put forward on phenomenological grounds in [18] and claimed to exist in the axial gauge in [16], but recently such a form of the gluon propagator in the infrared has been argued to be inconsistent [19].) In the Landau gauge the ghost sector has been completely neglected; thus it is much less straightforward to relate these results to physical observables. In section 6 we will discuss the comparison between the ERGE and SDE approach in more detail.

The paper is organized as follows: in section 2 we discuss the derivation of the ERGEs, and their relation to SDEs. In section 3 we present our truncated ansatz for the pure Yang-Mills action. In section 4 we discuss the technical procedure, and in section 5 our numerical results. Section 6 is devoted to conclusions and an outlook.

\section{ERGEs and SDEs}

The form of the ERGEs can most conveniently be derived assuming a path integral representation for the generating functional of the theory. To this end we restrict ourselves, for the time being, to the case of a single scalar field. It is straightforward to supplement the following equations with the appropriate minus signs, Lorentz and internal indices in the cases of fermions, vector fields and internal symmetries. To start with, the path integral formulation of the theory requires for its proper definition a regularization of the ultraviolet divergences. This will be implemented through a modification of the propagator, i.e. the term quadratic in the fields in the bare action. In addition we introduce an infrared cutoff $k$; the ERGEs will describe the variation of the generating functional with $k$, where the UV cutoff $\Lambda$ will be held fixed. (In the framework of the ERGEs, in particular in the application to gauge theories, one can consistently set $\Lambda=\infty$, see below). The UV and IR regularized generating functional of connected Green functions $\mathcal{G}_{k}^{\Lambda}(J)$ can then be represented as

$$
e^{-\mathcal{G}_{k}^{\Lambda}(J)}=\mathcal{N} \int \mathcal{D} \varphi e^{-\frac{1}{2}\left(\varphi, P_{0}^{-1} \varphi\right)-\Delta S_{k}^{\Lambda}-S_{i n t}(\varphi)+(J, \varphi)}
$$

where $S_{\text {int }}(\varphi)$ is independent of $k$. Here $(J, \varphi)$ etc. is a short-hand notation for

$$
(J, \varphi) \equiv \int \frac{d^{4} p}{(2 \pi)^{4}} J(p) \varphi(-p)
$$


$P_{0}^{-1}$ denotes the bare inverse propagator (e.g. $P_{0}^{-1}\left(p^{2}\right)=p^{2}$ in the case of a massless scalar field), $S_{\text {int }}$ the bare interaction, and

$$
\Delta S_{k}^{\Lambda}=\frac{1}{2}\left(\varphi, R_{k}^{\Lambda} \varphi\right)
$$

implements the UV and IR cutoffs. In the case of a massless field a convenient choice for $R_{k}^{\Lambda}$ is

$$
R_{k}^{\Lambda}\left(p^{2}\right)=p^{2} \frac{1-e^{-\frac{p^{2}}{\Lambda^{2}}}+e^{-\frac{p^{2}}{k^{2}}}}{e^{-\frac{p^{2}}{\Lambda^{2}}}-e^{-\frac{p^{2}}{k^{2}}}},
$$

such that the full propagator

$$
P_{k}^{\Lambda}=\left(P_{0}^{-1}+R_{k}^{\Lambda}\right)^{-1}=\frac{e^{-\frac{p^{2}}{\Lambda^{2}}}-e^{-\frac{p^{2}}{k^{2}}}}{p^{2}}
$$

has the desired properties: exponential decay in the UV, and finiteness in the IR.

The ERGE for $\mathcal{G}_{k}^{\Lambda}$ describes its variation with $k$, at fixed $\Lambda$. It can be obtained by differentiating eq. (2.1) with respect to $k$, and replacing $\varphi$ under the path integral by variations with respect to the sources. One finds

$$
\partial_{k} \mathcal{G}_{k}^{\Lambda}(J)=-\frac{1}{2} \int \frac{d^{4} q}{(2 \pi)^{4}} \partial_{k} R_{k}^{\Lambda}\left(q^{2}\right) \cdot\left\{\frac{\delta^{2} \mathcal{G}_{k}^{\Lambda}(J)}{\delta J(q) \delta J(-q)}-\frac{\delta \mathcal{G}_{k}^{\Lambda}(J)}{\delta J(q)} \frac{\delta \mathcal{G}_{k}^{\Lambda}(J)}{\delta J(-q)}\right\} .
$$

The effective action $\Gamma_{k}^{\Lambda}(\varphi)$ is defined by the Legendre transform of $\mathcal{G}_{k}^{\Lambda}(J)$,

$$
\Gamma_{k}^{\Lambda}(\varphi)=\mathcal{G}_{k}^{\Lambda}(J)+(J, \varphi)
$$

and it is convenient to define an effective action $\widehat{\Gamma}_{k}^{\Lambda}$ with the IR cutoff terms subtracted:

$$
\widehat{\Gamma}_{k}^{\Lambda}=\Gamma_{k}^{\Lambda}-\Delta S_{k}^{\Lambda}
$$

Inserting the Legendre transformation into eq. (2.6) one obtains the ERGE for $\widehat{\Gamma}_{k}^{\Lambda}$ :

$$
\partial_{k} \widehat{\Gamma}_{k}^{\Lambda}(\varphi)=\frac{1}{2} \int \frac{d^{4} q}{(2 \pi)^{4}} \partial_{k} R_{k}^{\Lambda}\left(q^{2}\right) \cdot\left(\frac{\delta^{2} \widehat{\Gamma}_{k}^{\Lambda}(\varphi)}{\delta \varphi(q) \delta \varphi(-q)}+R_{k}^{\Lambda}\left(q^{2}\right)\right)^{-1}
$$


The boundary condition of $\widehat{\Gamma}_{k}^{\Lambda}(\varphi)$ for $k \rightarrow \Lambda$ can be obtained from a careful consideration of the limit $P_{k}^{\Lambda} \rightarrow 0$ for $k \rightarrow \Lambda$ and reads

$$
\widehat{\Gamma}_{\Lambda}^{\Lambda}(\varphi)=\frac{1}{2}\left(\varphi,\left(P_{0}^{\Lambda}\right)^{-1} \varphi\right)+S_{\text {int }}(\varphi)
$$

On the other hand, for $k \rightarrow 0 \widehat{\Gamma}_{k}^{\Lambda}(\varphi)$ becomes the full quantum effective action of a theory, which is defined by a bare interaction $S_{\text {int }}(\varphi)$ and a fixed UV cutoff $\Lambda$. Since the integration of the ERGEs allows us to relate effective actions $\widehat{\Gamma}_{k}^{\Lambda}$ with different $k$, it can be used to compute $\widehat{\Gamma}_{0}^{\Lambda}$ in terms of $\widehat{\Gamma}_{\Lambda}^{\Lambda}$ related to $S_{\text {int }}$ by $(2.10)$.

For most applications in particle physics this is actually all we want: there always exists a scale $\Lambda$ (at least the Planck scale) beyond which we expect unknown new interactions or particles with masses larger than $\Lambda$. Physics beyond $\Lambda$, including quantum fluctuations involving momenta $p^{2}$ with $p^{2} \geq \Lambda^{2}$, will certainly affect the form of $S_{\text {int }}$ or the effective action $\widehat{\Gamma}_{\Lambda}^{\Lambda}$. Given an ansatz for $\widehat{\Gamma}_{\Lambda}^{\Lambda}$, however, we are generally interested in the effect of quantum fluctuations involving momenta with $0 \leq p^{2} \leq \Lambda^{2}$. If we integrate the ERGE for $\widehat{\Gamma}_{k}^{\Lambda}$ from $k=\Lambda$ down to $k=0$, these quantum fluctuations have been fully taken into account.

Next we turn to the relation between ERGEs and SDEs. To this end it is first convenient to write the ERGEs in terms of the generating functional (or partition function) $Z_{k}^{\Lambda}(J)$, where $Z_{k}^{\Lambda}(J)$ is given by

$$
Z_{k}^{\Lambda}(J)=e^{-\mathcal{G}_{k}^{\Lambda}(J)}
$$

and the r.h.side of eq. (2.11) by the path integral (2.1). The ERGE for $Z_{k}^{\Lambda}(J)$ reads

$$
\partial_{k} Z_{k}^{\Lambda}(J)=-\frac{1}{2} \int \frac{d^{4} q}{(2 \pi)^{4}} \partial_{k} R_{k}^{\Lambda}\left(q^{2}\right) \frac{\delta^{2} Z_{k}^{\Lambda}(J)}{\delta J(q) \delta J(-q)} .
$$

The full set of SDEs can also most conveniently be expressed in terms of $Z(J)[20]$. They follow from the vanishing of the path integral over a total derivative with respect to $\varphi$. Subsequently we will consider the SDEs in the presence of an UV cutoff $\Lambda$ and an IR cutoff $k$ as in (2.1). In terms of $Z_{k}^{\Lambda}(J)$ they read

$$
\left(J(p)-\left(P_{0}^{-1}\left(p^{2}\right)+R_{k}^{\Lambda}\left(p^{2}\right)\right) \frac{\delta}{\delta J(-p)}-\left.\frac{\delta S_{i n t}(\varphi)}{\delta \varphi(-p)}\right|_{\varphi=\frac{\delta}{\delta J}}\right) Z_{k}^{\Lambda}(J)=0
$$


After replacing $Z_{k}^{\Lambda}$ by $\mathcal{G}_{k}^{\Lambda}$ according to eq. (2.11), and $\mathcal{G}_{k}^{\Lambda}$ by $\Gamma_{k}^{\Lambda}$ according to eq. (2.7), one obtains the SDEs for the one-particle irreducible vertices, which are generally considered in the literature for $k=0$ and $\Lambda=\infty$.

Let us now denote the entire l.h.side of eq. (2.13) by $\Sigma_{k}^{\Lambda}(J)$. Next we evaluate the $k$-derivative of $\Sigma_{k}^{\Lambda}$. The $k$-derivative hits $R_{k}^{\Lambda}$ and $Z_{k}^{\Lambda}$, and we have to use the ERGE (2.12). The result can be written in the form

$$
\partial_{k} \Sigma_{k}^{\Lambda}=-\frac{1}{2} \int \frac{d^{4} q}{(2 \pi)^{4}} \partial_{k} R_{k}^{\Lambda}\left(q^{2}\right) \frac{\delta^{2} \Sigma_{k}^{\Lambda}}{\delta J(q) \delta J(-q)} .
$$

Thus we can make the following important statement: suppose that we managed to find a functional $Z_{k}^{\Lambda}(J)$, which satisfies the SDEs (2.13) for some value of $k$, identically in the sources $J$. Thus the corresponding quantity $\Sigma_{k}^{\Lambda}(J)$ vanishes. Now let us start to vary $k$. Since the r.h.side of eq. $(2.14)$ vanishes, $\Sigma_{k^{\prime}}^{\Lambda}$ will still vanish for $k^{\prime} \neq k$. (Here, of course, it has been used that $Z_{k}^{\Lambda}$ varies with $k$ according to the ERGE (2.12).) Thus we find that $Z_{k^{\prime}}^{\Lambda}$ still satisfies the $\operatorname{SDE}(2.13)$, with $k$ replaced by $k^{\prime}$. In other words, if we found a functional $Z_{k}^{\Lambda}$ which satisfies the $\operatorname{SDE}(2.13)$ for some value of $k$, and compute $Z_{k^{\prime}}^{\Lambda}$ by integrating the ERGE (2.12), $Z_{k^{\prime}}^{\Lambda}$ is guaranteed to satisfy the SDEs for all values for $k^{\prime}$. The same statement will hold for the other generating functionals $\mathcal{G}_{k}^{\Lambda}$ and $\Gamma_{k}^{\Lambda}$. Thus generating functionals which satisfy $k$-dependent SDEs can be named quasi-fixed points of the ERGEs ("quasi" because they still depend on $k$ ). In particular, for $k \rightarrow 0, \mathcal{G}_{0}^{\Lambda}$ and $\Gamma_{0}^{\Lambda}$ will satisfy the standard SDEs (without an infrared cutoff, but still an ultraviolet cutoff $\Lambda$ ) if such a quasi-fixed point is approached in the infrared.

Note that this property is independent of the form of $S_{\text {int }}$ in eq. (2.13): Any SDE with any form of $S_{\text {int }}$ in eq. (2.13) plays the role of a quasi-fixed point. (In particular, since we work in the presence of an UV cutoff $\Lambda, S_{\text {int }}$ can contain arbitrary higher dimensional interactions suppressed by powers of $\Lambda$.)

These formal arguments let us suspect, that results obtained by the integration of the ERGEs can be quite similar to results obtained by solving SDEs, although the formal arguments no longer hold in a strict sense as soon as the effective actions are approximated or truncated in some way.

Let us note, for later use, that on the r.h.sides of the ERGEs (2.6) and (2.9) we can replace $R_{k}^{\Lambda}\left(p^{2}\right)$ by $R_{k}\left(p^{2}\right)$, where $R_{k}\left(p^{2}\right)$ is defined by

$$
R_{k}\left(p^{2}\right)=R_{k}^{\Lambda=\infty}\left(p^{2}\right) .
$$


In the limit $\Lambda \rightarrow \infty$ the momentum integrations on the r.h.sides of (2.6) and (2.9) are still exponentially damped with the choice (2.4) for $R_{k}^{\Lambda}$. Accordingly we denote by $\mathcal{G}_{k}$ and $\widehat{\Gamma}_{k}$ the generating functionals, which satisfy the ERGEs with $R_{k}^{\Lambda}$ replaced by $R_{k}$. Of course we are still able to compute $\widehat{\Gamma}_{k=0}$ in terms of an ansatz for $\widehat{\Gamma}_{k=\Lambda}$. In physical terms UV divergences do not appear, since $\widehat{\Gamma}_{\Lambda}$ is already supposed to contain all quantum effects involving momenta with $p^{2} \geq \Lambda^{2}$. We just loose the possibility to formally equate $\widehat{\Gamma}_{\Lambda}$ with $S_{\text {int }}$ appearing in a path integral of the form of eq. (2.1), although a slightly different, more involved path integral representation for $\widehat{\Gamma}_{k}$ still exists [21]. From universality we expect in any case, that $\widehat{\Gamma}_{0}$ is independent of minor modifications of $\widehat{\Gamma}_{\Lambda}$, so one choice is a priori as good as the other. Thus, subsequently, we define a theory by the ERGEs (2.6) or (2.9), with $R_{k}^{\Lambda}$ replaced by $R_{k}$, and a finite ansatz for $\widehat{\Gamma}_{\Lambda}$ (or $\mathcal{G}_{\Lambda}$ ) at a fixed scale $\Lambda$.

\section{Yang-Mills Theories}

The application of ERGEs to gauge theories requires some treatment of the breaking of gauge invariance, which is introduced through the intermediate IR cutoff $k$. Here we use the method of imposing modified STIs [11, $13,14]$ in the formulation presented in $[13,14]$. In ref. [14], in particular, ERGEs for SU(3) Yang-Mills theory have already been integrated numerically within an approximation, where only marginal and relevant couplings in the effective action have been taken into account.

In the present paper we extend the form of the effective action considerably, namely we allow for an arbitrary momentum dependence of the gluon and ghost propagators. Again we consider the SU(3) Yang-Mills theory in four-dimensional Euclidean space-time, where the classical action reads

$$
\begin{aligned}
S= & \int \mathrm{d}^{4} x\left\{\frac{1}{4} F_{\mu \nu}^{a} F_{\mu \nu}^{a}+\frac{1}{2 \alpha} \partial_{\mu} A_{\mu}^{a} \partial_{\nu} A_{\nu}^{a}+\partial_{\mu} \bar{c}^{a}\left(\partial_{\mu} c^{a}+g f_{b c}^{a} A_{\mu}^{b} c^{c}\right)\right. \\
& \left.-K_{\mu}^{a}\left(\partial_{\mu} c^{a}+g f_{b c}^{a} A_{\mu}^{b} c^{c}\right)-L^{a} \frac{1}{2} g f_{b c}^{a} c^{b} c^{c}+\bar{L}^{a} \frac{1}{\alpha} \partial_{\mu} A_{\mu}^{a}\right\}
\end{aligned}
$$

with

$$
F_{\mu \nu}^{a}=\partial_{\mu} A_{\nu}^{a}-\partial_{\nu} A_{\mu}^{a}+g f_{b c}^{a} A_{\mu}^{b} A_{\nu}^{c} .
$$

We have included the usual gauge fixing and ghost parts and coupled external sources to the BRST variations 


$$
\begin{gathered}
\delta A_{\mu}^{a}=\left(\partial_{\mu} c^{a}+g f_{b c}^{a} A_{\mu}^{b} c^{c}\right) \zeta \\
\delta c^{a}=\frac{1}{2} g f_{b c}^{a} c^{b} c^{c} \zeta \\
\delta \bar{c}^{a}=-\frac{1}{\alpha} \partial_{\mu} A_{\mu}^{a} \zeta,
\end{gathered}
$$

where $\zeta$ is a Grassmann parameter. The invariance of $S$ at $\bar{L}=0$ under BRST transformations can then be expressed as

$$
\begin{gathered}
0=\left.\int \mathrm{d}^{4} x\left\{\delta A_{\mu}^{a} \frac{\delta S}{\delta A_{\mu}^{a}}+\delta c^{a} \frac{\delta S}{\delta c^{a}}+\delta \bar{c}^{a} \frac{\delta S}{\delta \bar{c}^{a}}\right\}\right|_{\bar{L}=0} \\
=\left.\zeta \int \mathrm{d}^{4} x\left\{\frac{\delta S}{\delta K_{\mu}^{a}} \frac{\delta S}{\delta A_{\mu}^{a}}-\frac{\delta S}{\delta L^{a}} \frac{\delta S}{\delta c^{a}}-\frac{\delta S}{\delta \bar{L}^{a}} \frac{\delta S}{\delta \bar{c}^{a}}\right\}\right|_{\bar{L}=0} .
\end{gathered}
$$

IR cutoffs in the gluon and ghost propagators will be introduced by adding a term of the form

$$
\Delta S_{k}=\frac{1}{2}\left(A_{\mu}^{a}, R_{k, \mu \nu} A_{\nu}^{a}\right)+\left(\bar{c}^{a}, \tilde{R}_{k} c^{a}\right)
$$

to the action. Explicit expressions for $R_{k, \mu \nu}$ and $\tilde{R}_{k}$ will be given below. The effective action

$$
\Gamma_{k}(A, c, \bar{c}, K, L, \bar{L}) \equiv \widehat{\Gamma}_{k}+\Delta S_{k}
$$

satisfies an ERGE of the form [13, 14]

$$
\begin{aligned}
\partial_{k} \widehat{\Gamma}_{k}= & \int \frac{d^{4} p}{(2 \pi)^{4}}\left\{\frac{1}{2} \partial_{k} R_{k, \mu \nu}\left(p^{2}\right)\left(\Gamma_{k}^{(2)}\right)_{A_{\nu}^{a}(-p), A_{\mu}^{a}(p)}^{-1}\right. \\
& \left.-\partial_{k} \tilde{R}_{k}\left(p^{2}\right)\left(\Gamma_{k}^{(2)}\right)_{-\bar{c}^{a}(-p), c^{a}(p)}^{-1}\right\} .
\end{aligned}
$$

Here $\left(\Gamma_{k}^{(2)}\right)_{A_{\mu}^{a}(-p), A_{\nu}^{b}(q)}^{-1}$ is the $\left(A_{\mu}^{a}(-p), A_{\nu}^{b}(q)\right)$-component of the inverse of the matrix $\Gamma_{k}^{(2)}=\delta^{2} \Gamma_{k} / \delta \bar{\varphi} \delta \varphi$ of second derivatives of $\Gamma_{k}$ with respect to the fields $\bar{\varphi}^{B}=\left\{A_{\mu}^{a}(-p),-\bar{c}^{a}(-p), c^{a}(-p)\right\}$ and $\varphi^{B}=\left\{A_{\mu}^{a}(p), c^{a}(p), \bar{c}^{a}(p)\right\}$, where the index of $\bar{\varphi}$ and $\varphi$ runs over the different fields, momenta, and Lorentz and gauge indices. For $k \rightarrow 0, \widehat{\Gamma}_{0}$ has to satisfy the STI (3.4). To this end $\widehat{\Gamma}_{k}$ has 
to satisfy, in particular at the starting point $k=\Lambda$, a modified STI of the form $[13,14]$

$$
\begin{gathered}
\left.\int \frac{d^{4} p}{(2 \pi)^{4}}\left\{\frac{\delta \widehat{\Gamma}_{k}}{\delta K_{\mu}^{a}(-p)} \frac{\delta \widehat{\Gamma}_{k}}{\delta A_{\mu}^{a}(p)}-\frac{\delta \widehat{\Gamma}_{k}}{\delta L^{a}(-p)} \frac{\delta \widehat{\Gamma}_{k}}{\delta c^{a}(p)}-\frac{\delta \widehat{\Gamma}_{k}}{\delta \bar{L}^{a}(-p)} \frac{\delta \widehat{\Gamma}_{k}}{\delta \bar{c}^{a}(p)}\right\}\right|_{\bar{L}=0} \\
=\int \frac{d^{4} p}{(2 \pi)^{4}} \sum_{B}\left[R_{k, \mu \nu}\left(p^{2}\right) \frac{\delta^{2} \widehat{\Gamma}_{k}}{\delta K_{\nu}^{a}(-p) \delta \varphi^{B}}\left(\Gamma_{k}^{(2)}\right)_{\bar{\varphi}^{B}, A_{\mu}^{a}(p)}^{-1}\right. \\
-\tilde{R}_{k}\left(p^{2}\right) \frac{\delta^{2} \widehat{\Gamma}_{k}}{\delta L^{a}(-p) \delta \varphi^{B}}\left(\Gamma_{k}^{(2)}\right)_{\bar{\varphi}^{B}, c^{a}(p)}^{-1} \\
\left.-\tilde{R}_{k}\left(p^{2}\right) \frac{\delta^{2} \widehat{\Gamma}_{k}}{\delta \bar{L}^{a}(-p) \delta \varphi^{B}}\left(\Gamma_{k}^{(2)}\right)_{\bar{\varphi}^{B}, \bar{c}^{a}(p)}^{-1}\right]\left.\right|_{\bar{L}=0} .
\end{gathered}
$$

The crucial point is that once eq. (3.8) is satisfied for $k=\Lambda$, it will be satisfied by $\widehat{\Gamma}_{k}$ for any $k<\Lambda$ provided $\widehat{\Gamma}_{k}$ is obtained from $\widehat{\Gamma}_{\Lambda}$ by integrating the ERGE. In particular $\widehat{\Gamma}_{k=0}$ will satisfy the standard STI (3.4), if the IR cutoff functions $R_{k, \mu \nu}$ and $\tilde{R}_{k}$ vanish identically for $k \rightarrow 0$.

Most importantly, $\widehat{\Gamma}_{k}$ at the starting point $k=\Lambda$ can thus not be identified with the classical action (3.1), but it has to contain symmetry breaking terms specified by the need to satisfy eq. (3.8). The origin of these symmetry breaking terms can be understood from the interpretation of $\widehat{\Gamma}_{\Lambda}$ as a quantum effective action, where momenta $p^{2}$ with $p^{2} \geq \Lambda^{2}$ have already been integrated out; the symmetry breaking induced by this "IR" cutoff $\Lambda$ then generates the symmetry breaking terms in $\widehat{\Gamma}_{\Lambda}$.

As stated above, our approach towards the integration of the ERGE (3.7) is based on an expansion in powers of the gluon and ghost fields. We are interested in the form of the gluon and ghost propagators, since a certain combination thereof (see below) determines the heavy quark potential in the quenched approximation. It is thus essential to allow for an arbitrary momentum dependence of the 2-point functions in our ansatz for the effective action.

Expanding the ERGE (3.7) in powers of fields one easily finds that the full 3-gluon, 4-gluon, ghost-gluon and 4-ghost vertices appear on the r.h.sides of the ERGEs for the gluon and ghost propagators. In the sequel we will refer to parts of the vertices with certain Lorentz and gauge index structures as "operators". One can distinguish two different kinds of such operators: 
a) "Independent" operators, which are not fixed in terms of the propagators or other operators by the STI. An example is the contribution to the 4-gluon vertex obtained from a term involving four powers of the field strength $F_{\mu \nu}^{a}$ in the effective action. (The r.h.side of the corresponding ERGE depends on the full 2-, 3-, 4-, 5- and 6-point functions.)

b) "Dependent" operators, which are fixed by the modified STI in terms of the propagators and operators of type a). In general eq. (3.8) yields a system of coupled non-linear integral equations for the dependent operators. Examples are the operators corresponding to those parts of the 3- and 4-gluon vertices, which are obtained by an expansion of the expression $F_{\mu \nu}^{a}\left(f\left(D^{2}\right) F_{\mu \nu}\right)^{a}$ in powers of the gauge field (see eq. (1.1)), and a gluonic mass term (which we do not consider as part of the gluon propagator in this context).

Since our goal is the determination of the propagators, the only important operators for our purpose are those, that contribute significantly to the ERGEs for the 2-point functions. In a first approximation, we will neglect the contributions of the operators of type a), which are the ones not determined by the STI. Such an approximation is only expected to hold for a limited range of scales $k$, and can to some extent be checked a posteriori (see below). In any case, it turns the ERGE (3.7) into a closed system of integro-differential equations for the propagators, which can be integrated numerically.

Furthermore, with the simpler ansatz used in ref. [14] it has proved to be permissible in a quantitative sense to determine the operators of type b) through the standard form of the STI (eq. (3.8) with a vanishing r.h.side) instead of using the modified STI, with the important exception of the gluon mass term. Again, this may only be true within a certain range of $k$. We will therefore proceed in the same way here, i.e. we assume that we can approximate the operators of type b) by the corresponding ones obtained from the standard STI, as far as their quantitative contribution to the flow of the 2-point functions is concerned.

In practical terms, this assumption implies a tremendous technical simplification in the determination of these operators. For example, for the 3and 4-gluon vertices we can use the expressions resulting from an expansion of $F_{\mu \nu}^{a}\left(f\left(D^{2}\right) F_{\mu \nu}\right)^{a}$ in powers of $A_{\mu}^{a}$ (apart from a momentum-dependent field 
renormalization, cf. the explicit expressions in the appendix). We consider it to be essential, however, to use the modified form of the STI to fix the gluon mass term, which is a truly relevant parameter of the effective action.

Let us stress that the approximation we employ for the operators of type b) does not imply that we are working with an effective action, which violates the modified STI. In principle we could, for every value of $k \neq 0$, determine the operators of type b) by solving the system of coupled non-linear integral equations given by eq. (3.8), so that the resulting effective action would be in accord with the modified STI. What we actually do is to approximate the contributions of the operators of type b) to the flow of the 2-point functions by the contributions of the corresponding operators obtained from the standard STI, in the same way as we neglect the contributions of the operators of type a).

It is important to note, that there exists a non-trivial quantitative check for the self-consistency of the approximations employed. It is based on the consistency of the ERGE with the $k$-derivative of the modified STI in the absence of approximations, and will be discussed in more detail in the next section. In particular, this self-consisteny condition will be used to determine the range of scales $k$, where we consider the approximations to be permissible.

The approximations employed here can be improved systematically by taking more and more operators of type a) into account, as well as by using the operators of type b) corresponding to a (partial or complete) solution of eq. (3.8). The purpose of the present paper is to see, which results for the heavy quark potential will be obtained within the "minimal" approach described above.

Let us now write down those terms in the $k$-dependent effective action, which we take into account on the r.h.sides of the ERGEs for the 2-point functions. In a slight abuse of notation, we will denote this part of the effective action by $\widehat{\Gamma}_{k}$. Firstly, we include the gluon and ghost $2-$ point functions themselves, which are given by the momentum-dependent functions $f_{1, k}$ and $f_{2, k}$, respectively, and where we take a mass term for the gluons into account. Since we will consider the Landau gauge later on, it is not necessary to introduce a further momentum-dependent function in the longitudinal part of the gluon 2-point function here. Secondly, we describe the 3-gluon, 4-gluon and ghost-gluon vertices of the type b) above (determined from the standard STI), which we take into account on the r.h.sides of the ERGEs for the $2-$ point functions. Finally we include the external source terms containing 
$K_{\mu}^{a}, L^{a}$ and $\bar{L}^{a}$, which are required to formulate the STI. These latter terms are constrained by two further identities satisfied by $\widehat{\Gamma}_{k}$, which can be shown to be invariant under the RG flow $[11,13,14]$ :

$$
\begin{gathered}
\partial_{\mu} \frac{\delta \widehat{\Gamma}_{k}}{\delta K_{\mu}^{a}}=\frac{\delta \widehat{\Gamma}_{k}}{\delta \bar{c}^{a}}, \\
\frac{\delta \widehat{\Gamma}_{k}}{\delta \bar{L}^{a}}=\frac{1}{\alpha} \partial_{\mu} A_{\mu}^{a} .
\end{gathered}
$$

Explicitly, then, our ansatz for $\widehat{\Gamma}_{k}$ reads

$$
\begin{gathered}
\widehat{\Gamma}_{k}(A, c, \bar{c}, K, L, \bar{L})= \\
\frac{1}{2} \int_{p_{1}, p_{2}} A_{\mu}^{a}\left(p_{1}\right)\left\{\left(p_{1}^{2} \delta_{\mu \nu}-p_{1 \mu} p_{1 \nu}\right) f_{1, k}\left(p_{1}^{2}\right)+\frac{p_{1 \mu} p_{1 \nu}}{\alpha}+m_{k}^{2} \delta_{\mu \nu}\right\} A_{\nu}^{a}\left(p_{2}\right) \\
+i g_{k} f_{b c}^{a} \int_{p_{1}, p_{2}, p_{3}} f_{3, \mu \nu \rho}\left(p_{1}, p_{2}, p_{3}\right) A_{\mu}^{a}\left(p_{1}\right) A_{\nu}^{b}\left(p_{2}\right) A_{\rho}^{c}\left(p_{3}\right) \\
+g_{k}^{2} f_{b c}^{a} f_{d e}^{a} \int_{p_{1}, \ldots, p_{4}} f_{4, \mu \nu \rho \sigma}\left(p_{1}, p_{2}, p_{3}, p_{4}\right) A_{\mu}^{b}\left(p_{1}\right) A_{\nu}^{c}\left(p_{2}\right) A_{\rho}^{d}\left(p_{3}\right) A_{\sigma}^{e}\left(p_{4}\right) \\
+\int_{p_{1}, p_{2}} \bar{c}^{a}\left(p_{1}\right) p_{1}^{2} f_{2, k}\left(p_{1}^{2}\right) c^{a}\left(p_{2}\right) \\
+i g_{k} f_{b c}^{a} \int_{p_{1}, p_{2}, p_{3}} \bar{c}^{a}\left(p_{1}\right) p_{1, \mu} f_{2, k}\left(p_{1}^{2}\right) f_{2, k}^{-1}\left(p_{2}^{2}\right) A_{\mu}^{b}\left(p_{2}\right) c^{c}\left(p_{3}\right) \\
+i \int_{p_{1}, p_{2}} K_{\mu}^{a}\left(p_{1}\right) p_{1 \mu} f_{2, k}\left(p_{1}^{2}\right) c^{a}\left(p_{2}\right) \\
-g_{k} f_{b c}^{a} \int_{p_{1}, p_{2}, p_{3}} K_{\mu}^{a}\left(p_{1}\right) f_{2, k}\left(p_{1}^{2}\right) f_{2, k}^{-1}\left(p_{2}^{2}\right) A_{\mu}^{b}\left(p_{2}\right) c^{c}\left(p_{3}\right) \\
-\frac{g_{k}}{2} f_{b c}^{a} \int_{p_{1}, p_{2}, p_{3}} L^{a}\left(p_{1}\right) c^{b}\left(p_{2}\right) c^{c}\left(p_{3}\right) \\
-\frac{i}{\alpha} \int_{p_{1}, p_{2}} \bar{L}^{a}\left(p_{1}\right) p_{1 \mu} A_{\mu}^{a}\left(p_{2}\right)
\end{gathered}
$$

where

$$
\int_{p_{1}, \ldots, p_{n}} \equiv \int \prod_{i=1}^{n}\left(\frac{d^{4} p_{i}}{(2 \pi)^{4}}\right) \cdot(2 \pi)^{4} \delta^{4}\left(\sum_{i=1}^{n} p_{i}\right)
$$


The $k$-dependent 3-gluon and 4-gluon vertex functions $f_{3, \mu \nu \rho}$ and $f_{4, \mu \nu \rho \sigma}$ are given in terms of the gluon propagator function $f_{1, k}$ and the ghost propagator function $f_{2, k}$ in the appendix.

In order to establish the connection with the heavy quark potential, we introduce quark fields in the effective action. In the limit where the quark mass tends to infinity, all quantum corrections corresponding to diagrams which contain inner quark lines, are naively suppressed by powers of the quark mass. On the other hand, the STI requires a momentum dependent quark-gluon vertex function, where the momentum dependence involves the function $f_{2, k}$ above. (Within the standard approach, the origin of this non-trivial contribution is due to UV divergent diagrams, which are not suppressed by the heavy quark mass.) After an eventual redefinition of the quark fields, such that their kinetic term is properly normalized, the standard STI constrains the quark-gluon coupling in the effective action to be of the form

$$
-g_{k} \lambda_{A B}^{a} \int_{p_{1}, p_{2}, p_{3}} \bar{\psi}^{A}\left(p_{1}\right) \gamma_{\mu} f_{2, k}^{-1}\left(p_{2}^{2}\right) A_{\mu}^{a}\left(p_{2}\right) \psi^{B}\left(p_{3}\right),
$$

where $\lambda$ denotes the $\mathrm{SU}(3)$ generators in the fundamental representation. Here we have implicitely used the $K c$-coupling from eq. (3.10) and the fact that the quantum corrections to the vertices, which couple external sources to the BRST-variations of the quark fields, are suppressed by powers of the quark mass in the Landau gauge.

Let us now consider the 2 -point function $V_{k}$ of the quark currents $J_{\mu}^{a}$, given by

$$
J_{\mu}^{a}=\lambda_{A B}^{a} \bar{\psi}^{A} \gamma_{\mu} \psi^{B} .
$$

In the heavy quark limit, the only contribution to $V_{k}$ comes from the dressed one-gluon exchange diagram, so from eq. (3.12) and the form of the gluon propagator we obtain the current 2 -point function

$$
V_{k}\left(p^{2}\right)=\frac{g_{k}^{2}}{p^{2} f_{1, k}\left(p^{2}\right) f_{2, k}^{2}\left(p^{2}\right)} .
$$

We have omitted the gluon mass term $m_{k}^{2}$ in our definition of $V_{k}\left(p^{2}\right)$. In principle we could have replaced the factor $p^{2} f_{1, k}\left(p^{2}\right)$ in the denominator of $V_{k}\left(p^{2}\right)$, which is due to the gluon propagator, by the factor involving $m_{k}^{2}$ and the infrared cutoff term. However, once the integration of the ERGE is pursued down to $k=0$, the modified STI (3.8) turns into the standard 
STI and enforces $m_{k=0}^{2}=0$, and the infrared cutoff term vanishes identically. Using eq. (3.14) to define $V_{k}\left(p^{2}\right)$, these effects have been anticipated, and $V_{k}\left(p^{2}\right)$ corresponds more closely to the physical function $V_{0}\left(p^{2}\right)$ already at finite $k$.

In the heavy quark limit $V_{k}\left(p^{2}\right)$ can be identified, up to trivial factors, with the Fourier transform of the potential between quarks in quenched QCD. Although we will compute the gluon and ghost propagator functions $f_{1, k}$ and $f_{2, k}$ individually by integrating the ERGEs, it is only the combination appearing in eq. (3.14), which has a physical meaning.

A priori it may seem that the functions $f_{1, k}$ and $f_{2, k}$ can be changed at will by redefining the gluon, ghost and anti-ghost fields in the form of multiplication with momentum dependent functions. We have checked explicitely, however, that the STI restricts the relations between the vertices and the two-point functions such that finally all possible field redefinitions cancel in the expression for $V_{k}\left(p^{2}\right)$.

As noted above and in refs. [11, 13,14], $m_{k}^{2}$ in $\widehat{\Gamma}_{k}$ is fixed in terms of the other parameters in $\widehat{\Gamma}_{k}$ by the non-vanishing r.h.side of eq. (3.8), where we use our ansatz (3.10) for $\widehat{\Gamma}_{k}$. After a consideration of terms $\sim A \cdot c$ of eq. (3.8), one arrives at a non-linear relation of the form

$$
m_{k}^{2}=\frac{g_{k}^{2}}{(4 \pi)^{2}} k^{2} \mathrm{ST}\left(k^{2}, m_{k}^{2}, f_{1, k}, f_{2, k}\right),
$$

where we have anticipated the Landau gauge and omitted the gauge parameter $\alpha$ on the r.h.side. The expression $\operatorname{ST}\left(k^{2}, \ldots\right)$ involves momentum integrations over a number of one-loop diagrams, in which the gluon and ghost propagators and hence the functions $f_{1, k}$ and $f_{2, k}$ appear, and will be given in the appendix. Generally eq. (3.15) can be solved for $m_{k}^{2}$ only numerically, but in any case $m_{k}^{2}$ is not an independent parameter of $\widehat{\Gamma}_{k}$.

To determine the flow of the coupling parameter $g_{k}$, we will use the ERGE for the $K c A$-vertex with vanishing momenta. Then it turns out, that in the Landau gauge $g_{k}$ actually does not run at all with $k$; it remains a constant, which will be specified once and for all at the starting point $k=\Lambda$. (It is straightforward, on the other hand, to define a "physical" running coupling in terms of the dressed one-gluon exchange diagram or the function $V_{k}$ of eq. (3.14), which runs with $k$ due to the running of $f_{1, k}$ and $f_{2, k}$. Within our approximation this running agrees to one-loop order with the usual perturbative running, as we have checked explicitely.) In the Landau gauge the 
independent running parameters in $\widehat{\Gamma}_{k}$ are thus only the two functions $f_{1, k}$ and $f_{2, k}$. The aim will be the determination of these quantities by integrating the ERGEs with suitable boundary conditions.

Finally we have to introduce and specify the IR cutoff term $\Delta S_{k}$ in eq. (3.5). If we demand a reasonable behaviour of the full propagators including the IR cutoff terms which appear on the r.h.side of the ERGE (3.7), we are lead to more sophisticated choices than an expression of the form of eq. (2.4) with $\Lambda \rightarrow \infty$. (Note that $R_{k, \mu \nu}$ and $\tilde{R}_{k}$ have to vanish identically for $k \rightarrow 0$ in order that the modified STI (3.8) turns into the standard one (3.4). To this end $\Lambda$ has to be put equal to $\infty$ inside $R_{k, \mu \nu}$ and $\tilde{R}_{k}$, which is perfectly consistent as discussed in sect. 2.) First, we do not want to generate any poles on the real axis through the introduction of $\Delta S_{k}$. This enforces a dependence of $R_{k, \mu \nu}$ and $\tilde{R}_{k}$ on the functions $f_{1, k}$ and $f_{2, k}$ such that they are proportional to $f_{1, k}$ and $f_{2, k}$, respectively. Now, however, it is no longer automatically guaranteed, that $\Delta S_{k}$ serves as an IR cutoff if, e.g., $f_{1, k}\left(p^{2}\right)$ vanishes for $p^{2} \rightarrow 0$ (as we might possibly expect). This problem is overcome by writing the functions $f_{1, k}$ and $f_{2, k}$ also in the exponents. Taking the presence of the gluon mass term $m_{k}^{2}$ into account, our choice of the cutoff functions thus reads

$$
\begin{aligned}
& R_{k, \mu \nu}\left(p^{2}\right)=\left(p^{2} f_{1, k}\left(p^{2}\right)+m_{k}^{2}\right) \frac{e^{-\left(p^{2} f_{1, k}\left(p^{2}\right)+m_{k}^{2}\right) / k^{2}}}{1-e^{-\left(p^{2} f_{1, k}\left(p^{2}\right)+m_{k}^{2}\right) / k^{2}}}\left(\delta_{\mu \nu}-\frac{p_{\mu} p_{\nu}}{p^{2}}\right) \\
&+\left(\frac{p^{2}}{\alpha}+m_{k}^{2}\right) \frac{e^{-\left(p^{2} / \alpha+m_{k}^{2}\right) / k^{2}}}{1-e^{-\left(p^{2} / \alpha+m_{k}^{2}\right) / k^{2}}} \frac{p_{\mu} p_{\nu}}{p^{2}} \\
& \tilde{R}_{k}\left(p^{2}\right)=p^{2} f_{2, k}\left(p^{2}\right) \frac{e^{-p^{2} f_{2, k}\left(p^{2}\right) / k^{2}}}{1-e^{-p^{2} f_{2, k}\left(p^{2}\right) / k^{2}}}
\end{aligned}
$$

In order to obtain the full propagators, these terms have to be added to the quadratic terms $\sim A \cdot A$ or $\sim \bar{c} \cdot c$ in the action $\widehat{\Gamma}_{k}$. Let us at this point remark a subtlety which has already been noted in ref. [14]: the modified STI (3.8) determines, after its expansion to $\mathcal{O}(A \cdot c)$, only the longitudinal part of the gluon propagator and requires the presence of a term of the form $m_{k}^{2} p_{\mu} p_{\nu} / p^{2}$. Then it is the condition of locality of the effective action, i.e. the need to cancel terms of the form $p_{\mu} p_{\nu} / p^{2}$, which requires the presence of the same mass term $m_{k}^{2}$ in the transverse part of the gluon propagator. Only after this consideration has been completed, we are allowed to approach the 
Landau gauge $\alpha=0$. In ref. [14] we had checked that $\alpha=0$ is preserved by the RG flow (and IR stable), and that the r.h.sides of the ERGEs remain finite. Since this choice simplifies the calculations considerably, we will work in the Landau gauge throughout the rest of this paper. The full gluon and ghost propagators, as derived from $\Gamma_{k}=\widehat{\Gamma}_{k}+\Delta S_{k}$, then read

$$
\frac{1-e^{-\left(p^{2} f_{1, k}\left(p^{2}\right)+m_{k}^{2}\right) / k^{2}}}{p^{2} f_{1, k}\left(p^{2}\right)+m_{k}^{2}}\left(\delta_{\mu \nu}-\frac{p_{\mu} p_{\nu}}{p^{2}}\right)
$$

and

$$
\frac{1-e^{-p^{2} f_{2, k}\left(p^{2}\right) / k^{2}}}{p^{2} f_{2, k}\left(p^{2}\right)},
$$

respectively.

\section{Procedure}

Let us now turn to the computation of $f_{1, k}$ and $f_{2, k}$ by integrating the ERGEs. The ERGE for $f_{1, k}$ can most easily be obtained by studying the terms quadratic in $A$ of the functional ERGE (3.7). Its diagrammatic form is shown in fig. 1 . Note that the $3-$ and $4-$ gluon vertices are more complicated than the ones of a classical Yang-Mills action and are given in the appendix. The form of the 3-gluon vertex coincides with the one used in the framework of SDEs [17] because it corresponds to a special solution of the standard STIs [22] (up to a momentum-dependent field redefinition); we have not been able to find the corresponding 4-gluon vertex elsewhere in the literature. The ERGE for $f_{2, k}$ is obtained from the terms $\sim \bar{c} c$ of eq. (3.7), and is also shown in diagrammatic form in fig. 1.

The crossed circles in fig. 1 denote insertions of $\partial_{k} R_{k, \mu \nu}$ resp. $\partial_{k} \tilde{R}_{k}$ according to the ERGE (3.7). Due to the complicated expressions (3.16) for these cutoff functions, i.e. their dependence on $m_{k}^{2}, f_{1, k}$ and $f_{2, k}$, the $k$-derivatives $\partial_{k}$ acting on $R_{k, \mu \nu}$ and $\tilde{R}_{k}$ have to be decomposed into partial derivatives $\partial / \partial k, \partial f_{1, k} / \partial k \cdot \partial / \partial f_{1, k}$, etc.

The system of ERGEs for $f_{1, k}$ and $f_{2, k}$ (in the Landau gauge), which is obtained from the diagrams of fig. 1, is thus of the form

$$
\begin{gathered}
\partial_{k} f_{1, k}=\frac{g_{\Lambda}^{2}}{(4 \pi)^{2}}\left(h_{1}^{0}\left(k^{2}, m_{k}^{2}, f_{1, k}, f_{2, k}\right)+\partial_{k} f_{1, k} * h_{1}^{1}\left(k^{2}, m_{k}^{2}, f_{1, k}, f_{2, k}\right)\right. \\
\left.+\partial_{k} f_{2, k} * h_{1}^{2}\left(k^{2}, m_{k}^{2}, f_{1, k}, f_{2, k}\right)+\partial_{k} m_{k}^{2} \cdot h_{1}^{3}\left(k^{2}, m_{k}^{2}, f_{1, k}, f_{2, k}\right)\right),
\end{gathered}
$$




$$
\begin{gathered}
\partial_{k} f_{2, k}=\frac{g_{\Lambda}^{2}}{(4 \pi)^{2}}\left(h_{2}^{0}\left(k^{2}, m_{k}^{2}, f_{1, k}, f_{2, k}\right)+\partial_{k} f_{1, k} * h_{2}^{1}\left(k^{2}, m_{k}^{2}, f_{1, k}, f_{2, k}\right)\right. \\
\left.+\partial_{k} f_{2, k} * h_{2}^{2}\left(k^{2}, m_{k}^{2}, f_{1, k}, f_{2, k}\right)+\partial_{k} m_{k}^{2} \cdot h_{2}^{3}\left(k^{2}, m_{k}^{2}, f_{1, k}, f_{2, k}\right)\right) .
\end{gathered}
$$

The evaluation of the expressions $h_{i}^{j}$ requires a numerical computation of the one-loop integrals appearing in fig. 1 . The $*$ denotes corresponding convolutions with respect to the loop momentum. It is evident from eqs. (4.1), that we still need the knowledge of $\partial_{k} m_{k}^{2}$ in order to determine $\partial_{k} f_{1, k}$ and $\partial_{k} f_{2, k} . \partial_{k} m_{k}^{2}$ can be obtained from the same diagrams in fig. 1, which are relevant for $\partial_{k} f_{1, k}$, in the limit of vanishing external momentum. Again this gives us an expression of the form

$$
\begin{aligned}
& \partial_{k} m_{k}^{2}=\frac{g_{\Lambda}^{2}}{(4 \pi)^{2}}\left(h_{3}^{0}\left(k^{2}, m_{k}^{2}, f_{1, k}, f_{2, k}\right)+\partial_{k} f_{1, k} * h_{3}^{1}\left(k^{2}, m_{k}^{2}, f_{1, k}, f_{2, k}\right)\right. \\
& \left.+\partial_{k} f_{2, k} * h_{3}^{2}\left(k^{2}, m_{k}^{2}, f_{1, k}, f_{2, k}\right)+\partial_{k} m_{k}^{2} \cdot h_{3}^{3}\left(k^{2}, m_{k}^{2}, f_{1, k}, f_{2, k}\right)\right) .
\end{aligned}
$$

Alternatively, we can obtain a similar expression by differentiating eq. (3.15) with respect to $k$. We thus have two different equations at our disposal to complete the system (4.1). In the absence of approximations, the two different completed systems would yield exactly the same results, as follows from the compatibility of the full equations (3.7) and (3.8). Since the r.h.sides of the eqs. (4.2) and (3.15) involve our ansatz (3.10) for $\widehat{\Gamma}_{k}$ rather than the full effective action, however, we obtain different results. (Nevertheless, they agree on the one-loop level [14].) The use of $\widehat{\Gamma}_{k}$ instead of the full effective action involves two different kinds of approximations related to the operators of type a) and b), respectively (see the previous section).

The compatibility of the two completed systems of equations considered above turns out to be sensitive to both kinds of approximations. As in ref. [14], we will use the difference between the corresponding results as a quantitative measure of the inadequacy of the approximations (for the respective scale $k$ ). Here we concentrate on the effect of the approximations on the gluon and ghost propagator functions: We will take a certain discrepancy (in fact $10 \%$ ) in the results for $\partial_{k} f_{1, k}$ or $\partial_{k} f_{2, k}$ at some value of the momentum as an indication that the approximations have a considerable influence on the contributions to the flow of $f_{1, k}$ or $f_{2, k}$. 
We are of course aware of the fact that the described consistency check merely yields a necessary condition for the validity of the approximation, not a sufficient one. There exists, however, another a-posteriori justification for our procedure: The results we obtain will show a remarkable degree of universality in the sense of ref. [23] (see the next section) as long as $k>k_{0}$, where the scale $k_{0}$ is determined from the above consistency condition. Since universality is only expected to hold when gauge symmetry is taken into account properly, and no significantly contributing operators are neglected, this property provides a strong indication of the consistency of our procedure.

In practice we will use the form of eq. (4.2) as derived from the ERGE (3.7) in order to complete the system of linear equations for $\partial_{k} f_{1, k}$ and $\partial_{k} f_{2, k}$. The other system of equations, with eq. (4.2) taken from the derivative of eq. (3.15), will be employed for the consistency check at every scale $k$.

Given the ERGEs for $f_{1, k}$ and $f_{2, k}$, we still need suitable boundary conditions in order to proceed with the numerical integration. These boundary conditions can be chosen such that $\widehat{\Gamma}_{\Lambda}$ resembles as close as possible the classical (bare) Yang-Mills action (3.1). One would thus choose, at $k=\Lambda$, $f_{1, \Lambda}\left(p^{2}\right)=f_{2, \Lambda}\left(p^{2}\right)=1$ and $g_{\Lambda}$ not too large in order to start in the perturbative regime. It is possible, however, to improve the form of the effective action at the starting point $k=\Lambda$. By definition, $\widehat{\Gamma}_{\Lambda}$ should include all quantum effects involving internal momenta $p^{2} \geq \Lambda^{2}$. On the one hand these are certainly small for a small coupling $g_{\Lambda}$, on the other hand it is fairly straightforward to include them to one-loop order. To this end one has to calculate the one-loop diagrams, which contribute to the gluon and ghost propagator, with IR cutoff functions $R_{k}$ as in eqs. (3.16) and $k=\Lambda$. (Inside these cutoff functions we can set, to one-loop order, $f_{1, k}\left(p^{2}\right)=f_{2, k}\left(p^{2}\right)=1$ and $m_{k}^{2}=0$ ). The diagrams have to be regularized in the UV (e.g. dimensionally) and renormalized such that, e.g., the renormalization conditions

$$
f_{1, \Lambda}(0)=1 \quad, \quad f_{2, \Lambda}(0)=1
$$

are satisfied. As a result one obtains

$$
\begin{gathered}
f_{1, \Lambda}\left(p^{2}\right)=1+\delta f_{1}^{1-\mathrm{loop}}\left(p^{2}\right) \\
f_{2, \Lambda}\left(p^{2}\right)=1+\delta f_{2}^{1-\mathrm{loop}}\left(p^{2}\right)
\end{gathered}
$$

where the one-loop contributions $\delta f_{i}^{1-\text { loop }}$ are given in the appendix. For 
large $p^{2}$ the functions $\delta f_{i}^{1-\text { loop }}$ behave asymptotically as

$$
\begin{aligned}
\delta f_{1}^{1-\text { loop }}\left(p^{2}\right) & \sim \frac{g_{\Lambda}^{2}}{(4 \pi)^{2}} \cdot \frac{13}{2} \ln \left(\frac{p^{2}}{\Lambda^{2}}\right), \\
\delta f_{2}^{1-\operatorname{loop}}\left(p^{2}\right) & \sim \frac{g_{\Lambda}^{2}}{(4 \pi)^{2}} \cdot \frac{9}{4} \ln \left(\frac{p^{2}}{\Lambda^{2}}\right) .
\end{aligned}
$$

What we have achieved now is an appropriate behaviour of these functions even for momenta $p^{2} \gg \Lambda^{2}$, far above the starting scale, provided $g_{\Lambda}^{2} /(4 \pi)^{2}$. $\ln \left(p^{2} / \Lambda^{2}\right) \ll 1$. Now $V_{k}\left(p^{2}\right)$ of eq. (3.14) has the appropriate logarithmic $p^{2}-$ dependence even for $p^{2} \gg \Lambda^{2}$, corresponding to a one-loop RG improvement of the "physical" coupling constant:

$$
V_{k=\Lambda}\left(p^{2} \gg \Lambda^{2}\right) \sim \frac{g_{\Lambda}^{2}}{p^{2}\left(1+\frac{11 g_{\Lambda}^{2}}{(4 \pi)^{2}} \ln \left(\frac{p^{2}}{\Lambda^{2}}\right)\right)} .
$$

As boundary conditions for the integration of the ERGEs we thus use propagator functions $f_{i, \Lambda}$ according to eqs. (4.4), the numerical solution of eq. (3.15) for $m_{\Lambda}^{2}$, and values for $g_{\Lambda}$ (as the only free parameter) from $g_{\Lambda}=2.0$ down to $g_{\Lambda}=1.4$.

It is clear that, in order to integrate the ERGEs (4.1) for the functions $f_{1, k}$ and $f_{2, k}$, numerical methods have to be employed. To this end we need a parametrization of these functions, which allows for a reliable fit for all values of the scale $k$. Since these functions always behave like $f_{i, k}\left(p^{2} \rightarrow\right.$ $\infty) \rightarrow 1+\delta f_{i}^{1-\text { loop }}$, the following parametrization turns out to be convenient:

$$
f_{i, k}\left(p^{2}\right)=1-\sum_{j} \frac{\alpha_{j}^{i}}{1+\gamma_{j}^{i} p^{2}}+\delta f_{i}^{1-\mathrm{loop}}\left(p^{2}\right)
$$

with up to 6 pairs of parameters $\alpha_{j}^{i}$, $\gamma_{j}^{i}$ for $i=1,2$, respectively.

Next we have to diagonalize eqs. (4.1) in the space of momenta $p^{2}$. To this end we evaluate the functions $h_{i}^{j}$ in eqs. (4.1) and (4.2) for a number of momenta $p^{2}=p_{j}^{2}$ with up to 35 different values for $p_{j}^{2}$ in the range $10^{-1} k_{0}^{2}$ to $10 \Lambda^{2}$, where $k_{0}^{2}$ is determined dynamically (see below). (It turns out to be convenient to space the momenta $p_{j}^{2}$ equally on a logarithmic scale.) By solving the system of linear algebraic equations we then obtain expressions for 
$\partial_{k} f_{i, k}\left(p_{j}^{2}\right)$ and $\partial_{k} m_{k}^{2}$. One step in the integration of the ERGE then amounts to a computation of

$$
f_{i, k+\Delta k}\left(p_{j}^{2}\right)=f_{i, k}\left(p_{j}^{2}\right)+\Delta k \cdot \partial_{k} f_{i, k}\left(p_{j}^{2}\right)
$$

with $|\Delta k / k|<3 \cdot 10^{-2}$, and a subsequent determination of the new fit parameters $\alpha_{j}^{i}, \gamma_{j}^{i}$ of eq. (4.7).

The new value of the parameter $m_{k}^{2}$ gets determined by a new numerical solution of eq. (3.15) rather than by the numerical integration of $\partial_{k} m_{k}^{2}$; since it is a relevant parameter (the only one), for $k^{2} \ll \Lambda^{2}$ accumulated theoretical and numerical errors would easily generate a $m_{k}^{2}$ of $\mathcal{O}\left(\Lambda^{2}\right)$ instead of $\mathcal{O}\left(k^{2}\right)$, as it should be according to eq. (3.15).

Of course we have varied many details of the numerical procedure in order to test the robustness of the final results. An upper limit of $\sim 35$ different values for $p_{j}^{2}$ is dictated by the finite amount of available computing time, and this also keeps us from choosing arbitrarily small values for the gauge coupling $g_{\Lambda}$ at the starting point $k^{2}=\Lambda^{2}$, since then the ERGEs would have to be integrated over arbitrarily many orders of magnitude of the scale $k^{2}$ in order to arrive at the physically interesting non-perturbative regime.

\section{Results}

Our results can most easily be represented in terms of a function $F_{k}\left(p^{2}\right)$,

$$
F_{k}\left(p^{2}\right)=\frac{1}{g_{\Lambda}^{2}} f_{1, k}\left(p^{2}\right) f_{2, k}^{2}\left(p^{2}\right)
$$

which is simply related to the potential $V_{k}\left(p^{2}\right)$ in eq. (3.14):

$$
V_{k}\left(p^{2}\right)=\frac{1}{p^{2} F_{k}\left(p^{2}\right)}
$$

In figs. 2-4 we show our results for $F_{k}\left(p^{2}\right)$ for a bare coupling $g_{\Lambda}=1.4$. We plotted this function for different values of $k^{2}, k^{2}=\Lambda^{2}, 10^{-1} \Lambda^{2}, 10^{-2} \Lambda^{2}$, $10^{-3} \Lambda^{2}, k^{2}=k_{0}{ }^{2}=7.43 \cdot 10^{-5} \Lambda^{2}$, as full lines, and for some still smaller value $k^{2}=\hat{k}^{2}=3.68 \cdot 10^{-5} \Lambda^{2}$ as a dashed line. $p^{2}$ is given in units of $\Lambda^{2}$; the maximal value of $p^{2}$ is $\Lambda^{2}, 10^{-1} \Lambda^{2}$ and, in order to resolve the small $p^{2}$ region, $10^{-2} \Lambda^{2}$ in figs. 2 to 4 , respectively. The curves with decreasing values at $p^{2}=0$ correspond to decreasing values of $k^{2}$. We see that, for 
small enough $k^{2}$ and small enough $p^{2}, F_{k}\left(p^{2}\right)$ approaches a form $\sim p^{2}$ near the origin, which corresponds to a $1 / p^{4}$-behaviour of the potential $V_{k}\left(p^{2}\right)$.

Just before $F_{k}\left(p^{2}\right)$ becomes 0 for $p^{2} \rightarrow 0$, however, the two different methods of evaluating $\partial_{k} m_{k}^{2}$ (cf. eq. (4.2) and the discussion below) lead to different results for $\partial_{k} f_{1, k}$ or $\partial_{k} f_{2, k}$, with a relative difference of $\sim 0.1$. We denoted the corresponding scale by $k_{0}$. For slightly smaller values of $k$ the equation for the gluon mass term $m_{k}^{2}(3.15)$ possesses no longer a solution for $m_{k}^{2}$.

The incompatibility of the RG flow with the modified STIs (3.8) below $k_{0}$ indicates, that our approximation becomes unreliable in this regime: Numerically different results for $\partial_{k} f_{1, k}$ or $\partial_{k} f_{2, k}$ show, that the neglected contributions play an important role on the r.h.side of the ERGE for the gluon or ghost propagator, respectively, and that our ansatz is too restrictive in this regime.

The gluon mass term $m_{k}^{2}$ itself, which was determined by eq. (3.15) to be of $\mathcal{O}\left(\Lambda^{2}\right)$ at the starting point $k=\Lambda$, does not show an unusual behaviour for $k \sim k_{0} . m_{k}^{2}$ is always of $\mathcal{O}\left(k^{2}\right)$ or, more precisely, $m_{k}^{2} \sim-0.6 \cdot k^{2}$ for $k \rightarrow k_{0}$.

Thus we cannot have confidence in the form of the RG flow for $k<$ $k_{0}$. However, generally the $\mathrm{RG}$ flow modifies $F_{k}\left(p^{2}\right)$ only for $p^{2} \lesssim k^{2}$ (cf. figs. 2-4), hence our result for $F_{k_{0}}$ is nevertheless trustworthy for $p^{2} \gtrsim k_{0}^{2}$. Our results also indicate that, within a more general parametrization of $\hat{\Gamma}_{k}$, $F_{k}(0)$ approaches 0 for $k^{2} \rightarrow 0$ : In figs. 2-4 we have shown the function $F_{k}\left(p^{2}\right)$ for some value $k^{2}=\hat{k}^{2}<k_{0}^{2}$ as a dashed line, and we see that the decrease of $F_{k}\left(p^{2}\right)$ at $p^{2}=0$ continues. For still smaller values of $k^{2}$, however, the integration of the system of differential equations becomes numerically unstable and does no longer allow to obtain reliable results.

In any case, already our results for $F_{k_{0}}\left(p^{2}\right)$ for $p^{2} \gtrsim k_{0}^{2}$ strongly indicate a form $\sim p^{2}$ near the origin, and the trustworthy range of $p^{2}$ may be sufficient for some phenomenological investigations involving, e.g., heavy quarks.

It might be interesting to get some feeling for the orders of magnitude of the different scales involved. Up to now all scales are only defined relative to the starting scale $\Lambda$. In order to relate this scale to a physical scale one can try to compare our result for $V_{k_{0}}\left(p^{2}\right)$, for $p^{2} \gtrsim k_{0}{ }^{2}$, with a phenomenological parametrization of this potential, which contains dimensionful parameters known in units of $\mathrm{MeV}$. A convenient form of such a parametrization is given 
by Richardson [24], which reads in our convention

$$
V_{R}\left(p^{2}\right)=\frac{48 \pi^{2}}{\left(33-2 N_{f}\right) p^{2} \ln \left(1+p^{2} / \Lambda_{R}^{2}\right)}
$$

with $\Lambda_{R} \cong 400 \mathrm{MeV}$, and for a rough comparison with our results (in pure $\mathrm{SU}(3)$ Yang-Mills theory) we may set $N_{f}=0$. In terms of our function $F_{k}\left(p^{2}\right)$ of eq. (5.1) this ansatz reads

$$
F_{R}\left(p^{2}\right)=\frac{11 \ln \left(1+p^{2} / \Lambda_{R}^{2}\right)}{16 \pi^{2}}
$$

We performed a fit of eq. (5.4) to our function $F_{k_{0}}\left(p^{2}\right)$ for the region of momenta $k_{0}^{2} \ll p^{2} \ll \Lambda^{2}$ to determine the ratio $\Lambda / \Lambda_{R}$. The result is shown in figs. 5 and 6 , where we plotted $F_{k_{0}}\left(p^{2}\right)$ as a full line and $F_{R}\left(p^{2}\right)$, eq. (5.4), as a dashed line, against $p^{2}$ in $\mathrm{GeV}^{2}$. The optimized relation between $\Lambda$ and $\Lambda_{R}$ is such that $\Lambda=12.1 \mathrm{GeV}$, which implies that the scale $k_{0}$, where our approximation ceases to be appropriate, is $\sim 104 \mathrm{MeV}$. One can see that our potential is surprisingly close to the phenomenological one of Richardson, both in the perturbative (fig. 5) and in the nonperturbative (fig. 6) regime. Significant deviations show up only for momenta $p^{2} \lesssim k_{0}^{2}$.

Let us now look at the dependence of our results on the bare coupling $g_{\Lambda}$. We find that also for larger values of $g_{\Lambda}, F_{k}(0)$ becomes small for small $k$, until our consistency condition indicates the inadequacy of the approximation at some scale $k_{0}$, with $k_{0}{ }^{2} / \Lambda^{2}=6.13 \cdot 10^{-4}, 2.69 \cdot 10^{-3}$, and $7.98 \cdot 10^{-3}$ for $g_{\Lambda}=1.6$, 1.8 and 2.0, respectively. In fig. 7 we compare the different results for $F_{k_{0}}\left(p^{2}\right)$ with $g_{\Lambda}=1.4$ (full line), $g_{\Lambda}=1.6$ (long-dashed line), $g_{\Lambda}=1.8$ (short-dashed line) and $g_{\Lambda}=2.0$ (dotted line). In each case we determined the physical value of the starting scale by a fit to eq. (5.4), and the results for $\Lambda$ are given by $12.1 \mathrm{GeV}, 4.8 \mathrm{GeV}, 2.6 \mathrm{GeV}$ and $1.7 \mathrm{GeV}$ for $g_{\Lambda}=1.4,1.6,1.8$ and 2.0 , respectively. From the values of $k_{0}^{2} / \Lambda^{2}$ given above, we then obtain the corresponding values for $k_{0}, k_{0}=104 \mathrm{MeV}, 119 \mathrm{MeV}, 134 \mathrm{MeV}$ and $151 \mathrm{MeV}$. We see that the curves in fig. 7 coincide remarkably well, which assures us that the numerical method we use for the integration of the ERGEs does not accumulate numerical errors. The approximate independence of the curves on $g_{\Lambda}$ or $\Lambda$ even for momenta larger than the respective starting scale is due to the one-loop improvement of the starting action $\widehat{\Gamma}_{\Lambda}$ (or of the functions $f_{1, \Lambda}$ and $f_{2, \Lambda}$ according to eq. (4.4)). In fig. 8 we show the same curves for 
$F_{k_{0}}\left(p^{2}\right)$ for the different values of $g_{\Lambda}$ or $\Lambda$, which have been obtained without the one-loop improvement.

Figs. 7 and 8 lead to a series of important observations: First, as we see most clearly from fig. 8, the curves are essentially unchanged compared to their form at the respective starting scale $\Lambda$ for momenta $p^{2} \gg \Lambda^{2}$, as they should be. In particular, the curve for $\Lambda=1.7 \mathrm{GeV}\left(g_{\Lambda}=2.0\right)$ in fig. 7 is given in the perturbative region essentially by the one-loop form (4.4), so comparison with, e.g., the curves for $\Lambda=12.1 \mathrm{GeV}\left(g_{\Lambda}=1.4\right)$ in both figures shows, that the integration of the ERGEs correctly reproduces the one-loop form in the perturbative domain. More importantly, we find universal behaviour in the non-perturbative domain: For momenta $p^{2} \ll \Lambda^{2}$, i.e. below the respective starting scales, all curves merge in one, as is most impressively demonstrated by fig. 8, and the form of the resulting curve does not depend on the boundary condition we use. This independence of physics at $p^{2} \ll \Lambda^{2}$ with respect to variations of the starting scale $\Lambda$ and to the inclusion of irrelevant operators at $\Lambda$ (as is the case for the one-loop improved boundary conditions) is what we call universality here, in accordance with ref. [23].

It is easy to see that the relation between $g_{\Lambda}$ and the starting scale $\Lambda$, which has been obtained empirically above, agrees with the one-loop running of $g_{\Lambda}$. On the other hand the running of $g_{\Lambda}$ with $\Lambda$ is not yet correct to twoloop accuracy. However, with our ansatz for $\widehat{\Gamma}_{k}$ only a subset of all possible two-loop diagrams is included within a perturbative expansion of the ERGEs, hence an agreement at this level would have been mere luck.

One may ask whether the behaviour of $F_{k_{0}}\left(p^{2}\right)$ for $p^{2} \rightarrow 0$ is due to the form of $f_{1, k_{0}}\left(p^{2}\right)$ or both $f_{1, k_{0}}\left(p^{2}\right)$ and $f_{2, k_{0}}\left(p^{2}\right)$. To this end we plot in fig. 9 both functions $f_{1, k_{0}}\left(p^{2}\right)$ and $f_{2, k_{0}}\left(p^{2}\right)$ (with $p^{2}$ rescaled as before) for $g_{\Lambda}=$ 1.4. We see that both functions become small for $p^{2} \rightarrow 0$, but it would be grossly misleading (and unphysical) to identify the gluon propagator function $\left(p^{2} f_{1}\left(p^{2}\right)\right)^{-1}$ with the heavy quark potential and to neglect the dependence of $V\left(p^{2}\right)$ of eq. (3.14) on $f_{2}\left(p^{2}\right)$, as it is done if the ghost part of the action is neglected, and the STIs are not properly taken into account.

\section{Discussion and Outlook}

In this paper we have performed the integration of the ERGEs for nonabelian gauge theories, with the bare action as the only input. We concentrated on pure SU(3) Yang-Mills theory (or quenched QCD), and the 
momentum dependence of the gluon and ghost propagators; these quantities determine the heavy quark potential.

The neglect of certain contributions on the r.h.sides of the ERGEs for the 2-point functions turned the system of ERGEs into a closed system of differential equations, which we integrated numerically. A priori the validity of the approximation is difficult to judge in the strong coupling regime, where it amounts to the neglect of contributions of the same order in the coupling constant as the ones maintained. Here the use of modified STIs plays an important role: Due to their validity parts of the $k$-dependent effective action can be determined in two different ways, namely either directly through these identities or by integrating the ERGEs. Without approximations both methods give the same results, but in the presence of approximations a possible difference between the corresponding results can be used to estimate the error induced by the approximations. In our case it was the gluon mass term, which was obtained in two different ways. We used a $10 \%$-deviation in $\partial_{k} f_{1, k}$ or $\partial_{k} f_{2, k}$, induced by the different methods to obtain $\partial_{k} m_{k}^{2}$, as an indication for the breakdown of our approximation near the corresponding scale $k_{0}$. Accordingly, our result for $V\left(p^{2}\right) \equiv V_{k_{0}}\left(p^{2}\right)$ is only consistent for $p^{2} \gtrsim k_{0}^{2}$, where we estimated $k_{0} \sim 100 \mathrm{MeV}$. Our main findings are the strong indication of a $1 / p^{4}$-behaviour of $V\left(p^{2}\right)$ in the trustworthy regime of $p^{2}$, and the fact that the full form of $V\left(p^{2}\right)$ happens to be close to the one proposed by Richardson [24].

In the second section of this paper we discussed the formal relation between ERGEs and SDEs, with the result that effective actions, which solve SDEs in the presence of an IR cutoff $k$, can be considered as quasi-fixed points of the ERGEs. Although this formal relation is no longer exact in the presence of truncations of effective actions, it is thus not astonishing, that the behaviour of the gluon propagator, which has been derived here using the ERGEs, has also been shown to be a solution to SDEs in the Landau gauge [17]. We believe, however, that the present method possesses a number of advantages compared to the formalism of the SDEs: Firstly, the regularization of both ultraviolet and infrared singularities in the context of the SDEs generates notorious technical difficulties, which require, to some extent, ad hoc prescriptions, the influence of which on the final result is difficult to control. The present method is, in contrast, free of problems related to UV or IR singularities by construction.

Secondly, a priori both methods require a suitable truncation of the ef- 
fective action. Within the present method, however, it is technically simpler to include more and more terms; for the present investigation, e.g., we already included the contributions of the 4-gluon vertex and the ghost fields, which were neglected in the SDE approach [17]. We have stressed that the detailed form of the dressed one-gluon exchange diagram between heavy quarks, which involves the ghost propagator function at the quark-gluon vertex due to the STIs, differs considerably from the form of the inverse gluon propagator alone (in the Landau gauge), which is not a physical quantity by itself. Moreover, it is technically feasible in our formalism to include further terms in the effective action without too much effort.

Finally, within the SDE approach one is only able to check the selfconsistency of a particular ansatz; it is practically impossible to prove rigorously, that the chosen ansatz is the only possible one. The integration of the ERGEs, on the other hand, always gives a unique answer, namely the one which is smoothly connected to the corresponding bare action, and to perturbation theory in the UV in the case of asymptotic freedom. Actually, our discussion in section 2 sheds some light on the cases where different solutions of the SDEs exist: on the one hand solutions of the SDEs can be considered as fixed points of the ERGEs for $k \rightarrow 0$, on the other hand it is generally not clear, whether one has obtained an IR stable or an UV stable fixed point. Since only the IR stable solutions can also be obtained by the integration of the ERGEs towards $k \rightarrow 0$, one is lead to the conclusion, that only these are actually physically relevant, which is certainly difficult to check within the SDE approach alone.

Many possibilities exist in order to extend the present approach in the future: firstly, still more terms can be included in the effective action, and collective fields, e.g. for $F_{\mu \nu}^{a} F_{\mu \nu}^{a}$, can be introduced along the lines of refs. $[7,8]$ in order to reach renormalization scales $k$ below $k_{0}$, and to get a still better understanding of pure Yang-Mills theory free of systematic uncertainties for $p^{2}<k_{0}^{2}$. Secondly, the quark sector of QCD can be introduced as well. First efforts in this direction have already been made in ref. [8]; there, however, the effects of the gluons have been guessed and parametrized in a phenomenological manner. Now the integration of the ERGEs for the full quark gluon system is within reach (possibly along the lines proposed in ref. [25]), which will allow to study many phenomenologically interesting systems like heavy $q \bar{q}$-bound states, $q \bar{q}$-condensates and the meson sector on the basis of just the bare QCD Lagrangian. 


\section{Appendix}

In this appendix we list some of the functions which are needed in the actual computations. We begin with the 3- and 4-gluon vertices employed

in our ansatz (3.10) for $\widehat{\Gamma}_{k}$. They are chosen in such a way as to fulfill the standard STIs in a "minimal" fashion. Explicitly they read

$$
\begin{gathered}
f_{3, \mu \nu \rho}(p, q, r)=-\frac{1}{6 f_{2, k}\left(p^{2}\right) f_{2, k}\left(q^{2}\right) f_{2, k}\left(r^{2}\right)} \times \\
\left\{\left(q_{\mu} f_{g, k}\left(q^{2}\right)-r_{\mu} f_{g, k}\left(r^{2}\right)\right) \delta_{\nu \rho}+\left(r_{\nu} f_{g, k}\left(r^{2}\right)-p_{\nu} f_{g, k}\left(p^{2}\right)\right) \delta_{\rho \mu}\right. \\
+\left(p_{\rho} f_{g, k}\left(p^{2}\right)-q_{\rho} f_{g, k}\left(q^{2}\right)\right) \delta_{\mu \nu} \\
+\left(q_{\mu}-r_{\mu}\right) \frac{f_{g, k}\left(q^{2}\right)-f_{g, k}\left(r^{2}\right)}{q^{2}-r^{2}}\left(r_{\nu} q_{\rho}-q \cdot r \delta_{\nu \rho}\right) \\
+\left(r_{\nu}-p_{\nu}\right) \frac{f_{g, k}\left(r^{2}\right)-f_{g, k}\left(p^{2}\right)}{r^{2}-p^{2}}\left(p_{\rho} r_{\mu}-r \cdot p \delta_{\rho \mu}\right) \\
\left.+\left(p_{\rho}-q_{\rho}\right) \frac{f_{g, k}\left(p^{2}\right)-f_{g, k}\left(q^{2}\right)}{p^{2}-q^{2}}\left(q_{\mu} p_{\nu}-p \cdot q \delta_{\mu \nu}\right)\right\}
\end{gathered}
$$

and

$$
\begin{gathered}
f_{4, \mu \nu \rho \sigma}(p, q, r, s)=\frac{1}{4 f_{2, k}\left(p^{2}\right) f_{2, k}\left(q^{2}\right) f_{2, k}\left(r^{2}\right) f_{2, k}\left(s^{2}\right)} \times \\
\left\{f_{g, k}\left((p+q)^{2}\right) \delta_{\mu \rho} \delta_{\nu \sigma}-2 \frac{f_{g, k}\left(p^{2}\right)-f_{g, k}\left(s^{2}\right)}{p^{2}-s^{2}}\left(p_{\sigma} s_{\mu}-p \cdot s \delta_{\sigma \mu}\right) \delta_{\nu \rho}\right. \\
-2 \frac{f_{g, k}\left((p+q)^{2}\right)-f_{g, k}\left(p^{2}\right)}{(p+q)^{2}-p^{2}}\left(2 p_{\nu}+q_{\nu}\right)\left(p_{\rho} \delta_{\sigma \mu}-p_{\sigma} \delta_{\mu \rho}\right) \\
+\frac{2}{(r+s)^{2}-s^{2}}\left(\frac{f_{g, k}\left(p^{2}\right)-f_{g, k}\left((r+s)^{2}\right)}{p^{2}-(r+s)^{2}}-\frac{f_{g, k}\left(p^{2}\right)-f_{g, k}\left(s^{2}\right)}{p^{2}-s^{2}}\right) \\
\left.\times\left(2 p_{\nu}+q_{\nu}\right)\left(2 s_{\rho}+r_{\rho}\right)\left(p_{\sigma} s_{\mu}-p \cdot s \delta_{\sigma \mu}\right)\right\}
\end{gathered}
$$

where

$$
f_{g, k}\left(p^{2}\right)=f_{1, k}\left(p^{2}\right) f_{2, k}^{2}\left(p^{2}\right)
$$


Observe that we have not symmetrized the expression (A.2) for the fourgluon vertex with respect to momenta and indices, in order to keep it to a reasonable length.

Next we turn to the modified STI for the gluon mass term, eq. (3.15). In diagrammatic form it is given by a number of one-loop diagrams. The integrations over the angular variables of the loop momenta can be performed, and one is left with integrals over $t \equiv q^{2}$. After a careful consideration of the limit $\alpha \rightarrow 0$, corresponding to the Landau gauge, the following expression for the function ST results:

$$
\begin{gathered}
\operatorname{ST}\left(k^{2}, m_{k}^{2}, f_{1, k}, f_{2, k}\right)= \\
\frac{3}{4 f_{2, k}^{2}(0)} \int_{0}^{\infty} d t \frac{t}{k^{2}}\left\{-e^{-t f_{2, k}(t) / k^{2}}\left[3 f_{1, k}(t) \frac{1-e^{-\left(t f_{1, k}(t)+m_{k}^{2}\right) / k^{2}}}{t f_{1, k}(t)+m_{k}^{2}}\right.\right. \\
\left.+\left(3-4 \frac{t}{k^{2}} \frac{\partial}{\partial t}\left(t f_{2, k}(t)\right)\right) f_{2, k}(t) \frac{1-e^{-t f_{2, k}(t) / k^{2}}}{t f_{2, k}(t)}\right] \\
+3 e^{-\left(t f_{1, k}(t)+m_{k}^{2}\right) / k^{2}}\left[\left(4-4 \frac{t}{k^{2}} \frac{\partial}{\partial t}\left(t f_{1, k}(t)\right)\right) f_{1, k}(t) \frac{1-e^{-\left(t f_{1, k}(t)+m_{k}^{2}\right) / k^{2}}}{t f_{1, k}(t)+m_{k}^{2}}\right. \\
\left.+f_{2, k}(t) \frac{1-e^{-t f_{2, k}(t) / k^{2}}}{t f_{2, k}(t)}\right]-6 \frac{e^{-\left(t f_{1, k}(t)+m_{k}^{2}\right) / k^{2}}}{f_{2, k}^{2}(t)} t \frac{\partial}{\partial t}\left(f_{1, k}(t) f_{2, k}^{2}(t)\right) \times \\
{\left[\left(1+\frac{t}{k^{2}} \frac{\partial}{\partial t}\left(t f_{1, k}(t)\right)-\frac{2}{f_{2, k}(t)} \frac{\partial}{\partial t}\left(t f_{2, k}(t)\right)\right) \frac{1-e^{-\left(t f_{1, k}(t)+m_{k}^{2}\right) / k^{2}}}{t f_{1, k}(t)+m_{k}^{2}}\right.} \\
\left.\left.-t \frac{\partial}{\partial t}\left(t f_{1, k}(t)\right) \frac{1-\left(1+\left(t f_{1, k}(t)+m_{k}^{2}\right) / k^{2}\right) e^{-\left(t f_{1, k}(t)+m_{k}^{2}\right) / k^{2}}}{\left(t f_{1, k}(t)+m_{k}^{2}\right)^{2}}\right]\right\} .
\end{gathered}
$$

Finally we give the one-loop expressions $\delta f_{i}^{1-\text { loop }}$ for the gluon and ghost 2 -point functions as needed in (4.4) for the improved boundary conditions. They are calculated with the IR cutoff functions $R_{k}$ from eqs. (3.16) (with $f_{1, k}$ and $f_{2, k}$ set equal to one and $m_{k}^{2}=0$ ) and obey the renormalization conditions (4.3). The results are, again in the Landau gauge,

$$
\delta f_{1}^{1 \text {-loop }}\left(p^{2}\right)=\frac{3 g_{\Lambda}^{2}}{(4 \pi)^{2}}\left\{\frac{13}{6}(\ln (x / 2)+C)-\frac{131}{144}+\frac{27 x^{2}+88 x-20}{24 x^{3}}\right.
$$




$$
\begin{gathered}
-\frac{x^{4}-8 x^{3}+9 x^{2}-20 x-6}{12 x^{3}} e^{-x}+\frac{x^{4}-16 x^{3}-16 x^{2}-56 x+4}{12 x^{3}} e^{-x / 2} \\
\left.-\frac{x^{2}-7 x}{12} \operatorname{Ei}(-x)+\frac{x^{2}-14 x-52}{24} \operatorname{Ei}(-x / 2)\right\}\left.\right|_{x=\frac{p^{2}}{\Lambda^{2}}}
\end{gathered}
$$

and

$$
\begin{gathered}
\delta f_{2}^{1-\operatorname{loop}}\left(p^{2}\right)=\frac{3 g_{\Lambda}^{2}}{(4 \pi)^{2}}\left\{\frac{3}{4}(\ln (x / 2)+C)-\frac{3}{8}\right. \\
+\frac{3 x+2}{4 x^{2}}+\frac{x^{2}-x+2}{4 x^{2}} e^{-x}-\frac{x^{2}+x+2}{2 x^{2}} e^{-x / 2} \\
\left.+\frac{x}{4} \operatorname{Ei}(-x)-\frac{x+3}{4} \operatorname{Ei}(-x / 2)\right\}\left.\right|_{x=\frac{p^{2}}{\Lambda^{2}}}
\end{gathered}
$$

where $C$ denotes Euler's constant, $C \cong 0.577216$. The exponential integral function Ei is defined by

$$
\operatorname{Ei}(-x)=\int_{-\infty}^{-x} d t \frac{e^{t}}{t}
$$

for $x>0$.

\section{References}

[1] C. D. Roberts and A. G. Williams, Progress in Particle and Nuclear Physics 33 (1994) 477.

[2] K. G. Wilson and I. Kogut, Phys. Rep. 12 (1974) 75; F. Wegner, in Phase Transitions and Critical Phenomena, Vol. 6, eds. C. Domb and M. Green (Academic Press, NY 1975).

[3] J. Polchinski, Nucl. Phys. B231 (1984) 269; for recent references see J. Comellas, Y. Kubyshin and E. Moreno, FTUAM preprint 96/2, hep th/9601112.

[4] F. Wegner and A. Houghton, Phys. Rev. A8 (1973) 401; J. F. Nicoll, T. S. Chang and H. E. Stanley, Phys. Rev. Lett. 33 (1974) 540; A. Hasenfratz and P. Hasenfratz, Nucl. Phys. B270 (1986) 687. 
[5] S. Weinberg, in Proceedings of the 1976 International School of Subnuclear Physics, Erice (ed. A. Zichichi).

[6] U. Ellwanger, Z. Phys. C62 (1994) 503.

[7] U. Ellwanger, Z. Phys. C58 (1993) 679.

[8] U. Ellwanger and C. Wetterich, Nucl. Phys. B423 (1994) 137; D. Jungnickel and C. Wetterich, Phys. Rev. D53 (1996) 5142; D. Jungnickel, Heidelberg preprint HD-THEP-95-40, hep-ph/9509293.

[9] B. Warr, Ann. Phys. 183 (1988) 1 and ibid. 59.

[10] G. Keller and C. Kopper, Phys. Lett. B273 (1991) 323; G. Keller, Helv. Phys. Acta 66 (1993) 453.

[11] C. Becchi, in Elementary Particles, Field Theory and Statistical Mechanics, eds. M. Bonini, G. Marchesini and E. Onofri, Parma University 1993; M. Bonini, M. D'Attanasio and G. Marchesini, Nucl. Phys. B418 (1994) 81, Nucl. Phys. B421 (1994) 429, Nucl. Phys. B437 (1995) 163, Phys. Lett. B346 (1995) 87; M. D'Attanasio and T. Morris, hepth/9602156.

[12] M. Reuter and C. Wetterich, Nucl. Phys. B417 (1994) 181, ibid. B427 (1994) 291, Phys. Lett. B334 (1994) 412, Heidelberg preprint HDTHEP-94-39, hep-th/9411227.

[13] U. Ellwanger, Phys. Lett. B335 (1994) 364.

[14] U. Ellwanger, M. Hirsch and A. Weber, Z. Phys. C69 (1996) 687.

[15] M. Baker, J. S. Ball and F. Zachariasen, Nucl. Phys. B186 (1981) 531, ibid. B186 (1981) 560; A. I. Alekseev, Yad. Fiz. 33 (1981) 516; W. J. Schoenmaker, Nucl. Phys. B194 (1982) 535; F. Paccanoni, Nuovo Cim. A74 (1983) 267.

[16] J. R. Cudell and D. A. Ross, Nucl. Phys. B194 (1991) 247.

[17] S. Mandelstam, Phys. Rev. D20 (1979) 3223; U. Bar-Gadda, Nucl. Phys. B163 (1980) 312; N. Brown and M. K. Pennington, Phys. Lett. B202 (1988) 257, Phys. Rev. D39 (1989) 2723. 
[18] P. V. Landshoff and O. Nachtmann, Z. Phys. C39 (1989) 405.

[19] K. Büttner and M. R. Pennington, Phys. Rev. D52 (1995) 5220.

[20] C. Itzykson and J.-B. Zuber, Quantum Field Theory (McGraw-Hill, NY 1980).

[21] A. Weber, PhD Thesis, Univ. Heidelberg 1995.

[22] S. K. Kim and M. Baker, Nucl. Phys. B164 (1980) 152; J. S. Ball and J.-W. Chiu, Phys. Rev. D22 (1980) 2550.

[23] R. D. Ball and R. S. Thorne, Ann. Phys. 236 (1994) 117.

[24] J. L. Richardson, Phys. Lett. B82 (1979) 272.

[25] C. Wetterich, Heidelberg preprint HD-THEP-95-47.

\section{Figure captions}

Figure 1: Diagrammatic form of the ERGEs for the ghost propagator function $f_{2, k}$ and the gluon propagator function $f_{1, k}$. Internal curly lines denote the full gluon propagator, internal dotted lines the full ghost propagator and full points the full vertices from the ansatz (3.10) for $\widehat{\Gamma}_{k}$. The crossed circles denote insertions of $\partial_{k} R_{k, \mu \nu}$ resp. $\partial_{k} \tilde{R}_{k}$.

Figure 2: Results for the $\mathrm{k}$-dependent function $F_{k}\left(p^{2}\right)(5.1)$, which is related to the potential $V_{k}\left(p^{2}\right)$ through (5.2), for different values of $k^{2}$, $k^{2}=\Lambda^{2}, 10^{-1} \Lambda^{2}, 10^{-2} \Lambda^{2}, 10^{-3} \Lambda^{2}, k^{2}=k_{0}{ }^{2}=7.43 \cdot 10^{-5} \Lambda^{2}$, as full lines, and for $k^{2}=\hat{k}^{2}=3.68 \cdot 10^{-5} \Lambda^{2}$ as a dashed line. The bare coupling $g_{\Lambda}$ is $g_{\Lambda}=1.4$, and $p^{2}$ is given in units of $\Lambda^{2}$. The curves with decreasing values at $p^{2}=0$ correspond to decreasing values of $k^{2}$.

Figure 3: As in fig. 2, with a maximal value of $p^{2}=10^{-1} \Lambda^{2}$.

Figure 4: As in fig. 2, with a maximal value of $p^{2}=10^{-2} \Lambda^{2}$ in order to resolve the small $p^{2}$ region. 
Figure 5: Fit of our result for $F_{k_{0}}\left(p^{2}\right)$ (full line) to the parametrization of Richardson [24] (dashed line), with an optimized relation between $\Lambda$ and $\Lambda_{R} \cong 400 \mathrm{MeV}$ such that $\Lambda=12.1 \mathrm{GeV}$.

Figure 6: As in fig. 5, with a maximal value of $p^{2}=4 \mathrm{GeV}^{2}$.

Figure 7: Results for $F_{k_{0}}\left(p^{2}\right)$ for different values of the bare coupling $g_{\Lambda}=$ 1.4 (full line), $g_{\Lambda}=1.6$ (long-dashed line), $g_{\Lambda}=1.8$ (short-dashed line) and $g_{\Lambda}=2.0$ (dotted line). In each case we determined the physical value of the starting scale $\Lambda$ by a fit to the parametrization of Richardson, eq. (5.4).

Figure 8: As in fig. 7, but without the one-loop improvement of the propagator functions $f_{i, k}$ at the starting scale $\Lambda$.

Figure 9: Results for the propagator functions $f_{1, k_{0}}$ (full line) and $f_{2, k_{0}}$ (dashed line) for $g_{\Lambda}=1.4$. 
$\partial_{k} \quad \rightarrow \cdots \cdots \cdots \cdots=$

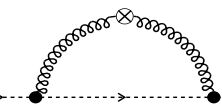

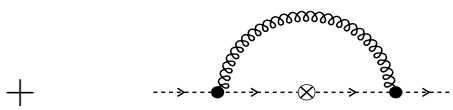

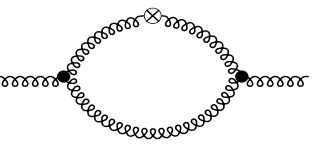

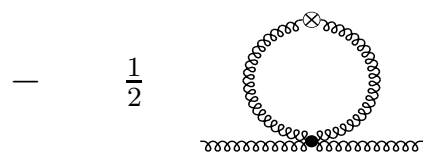
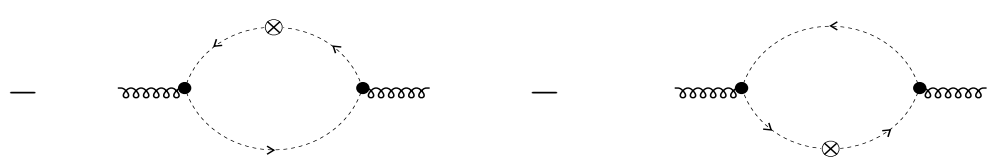

Figure 1

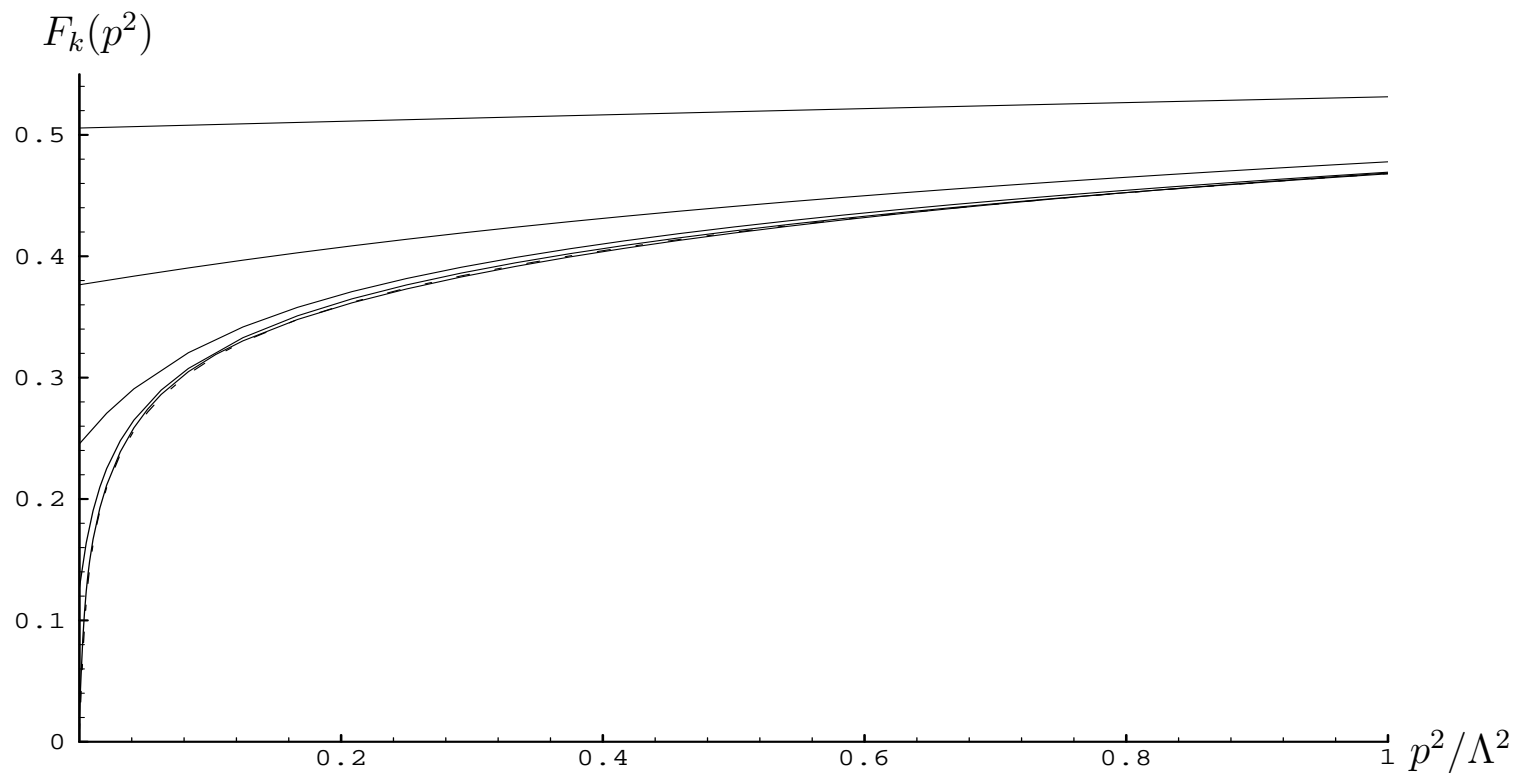

Figure 2 


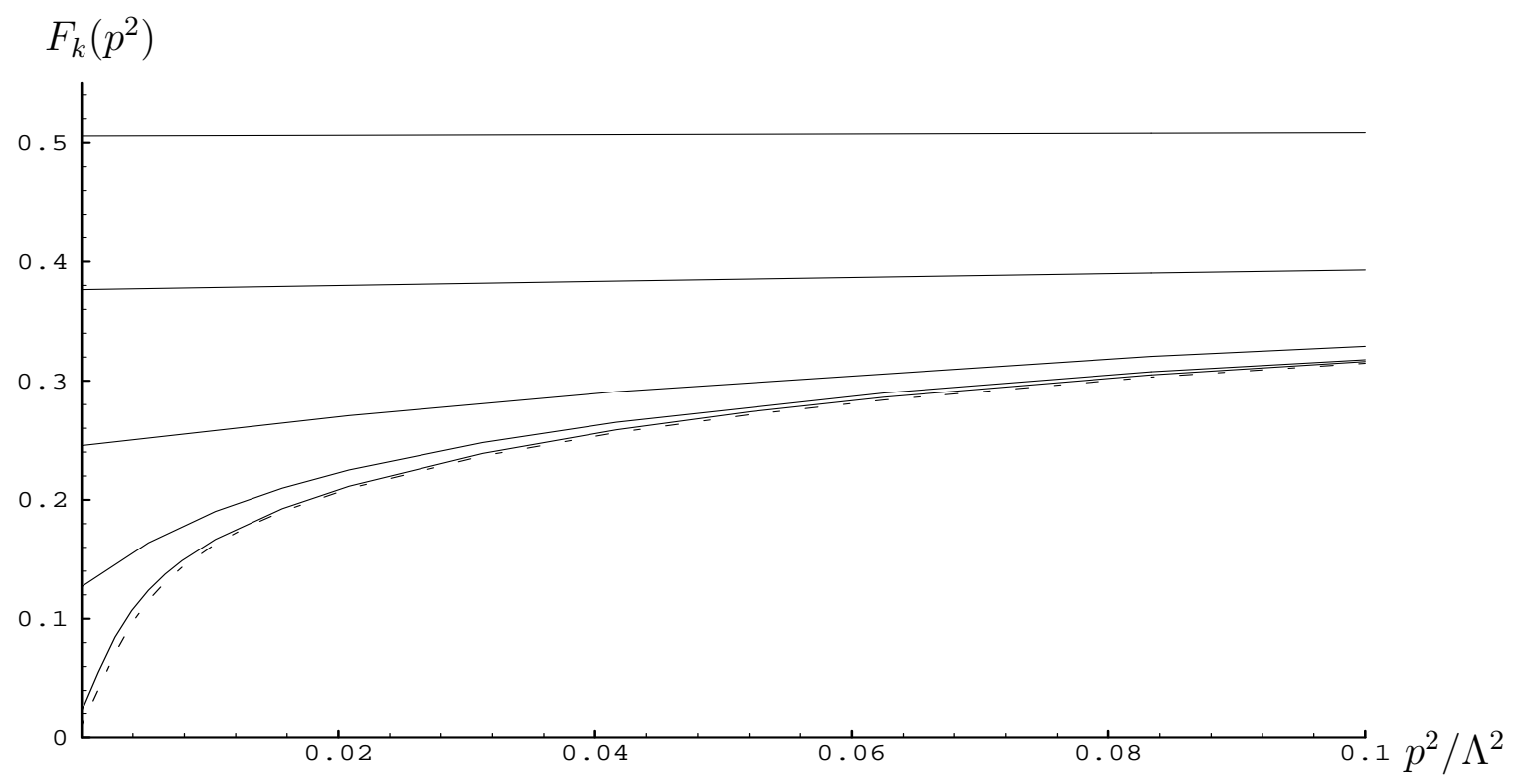

Figure 3

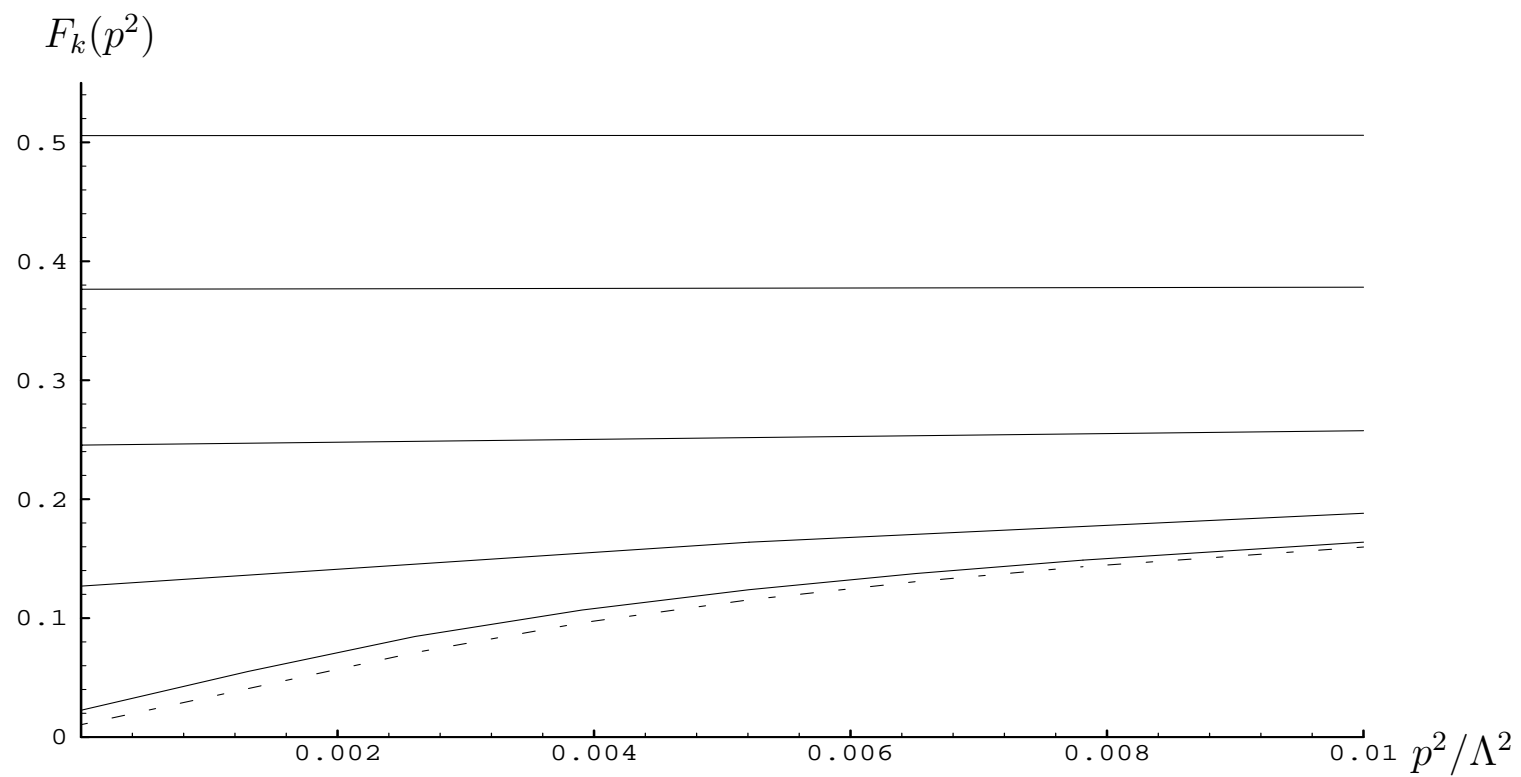

Figure 4 


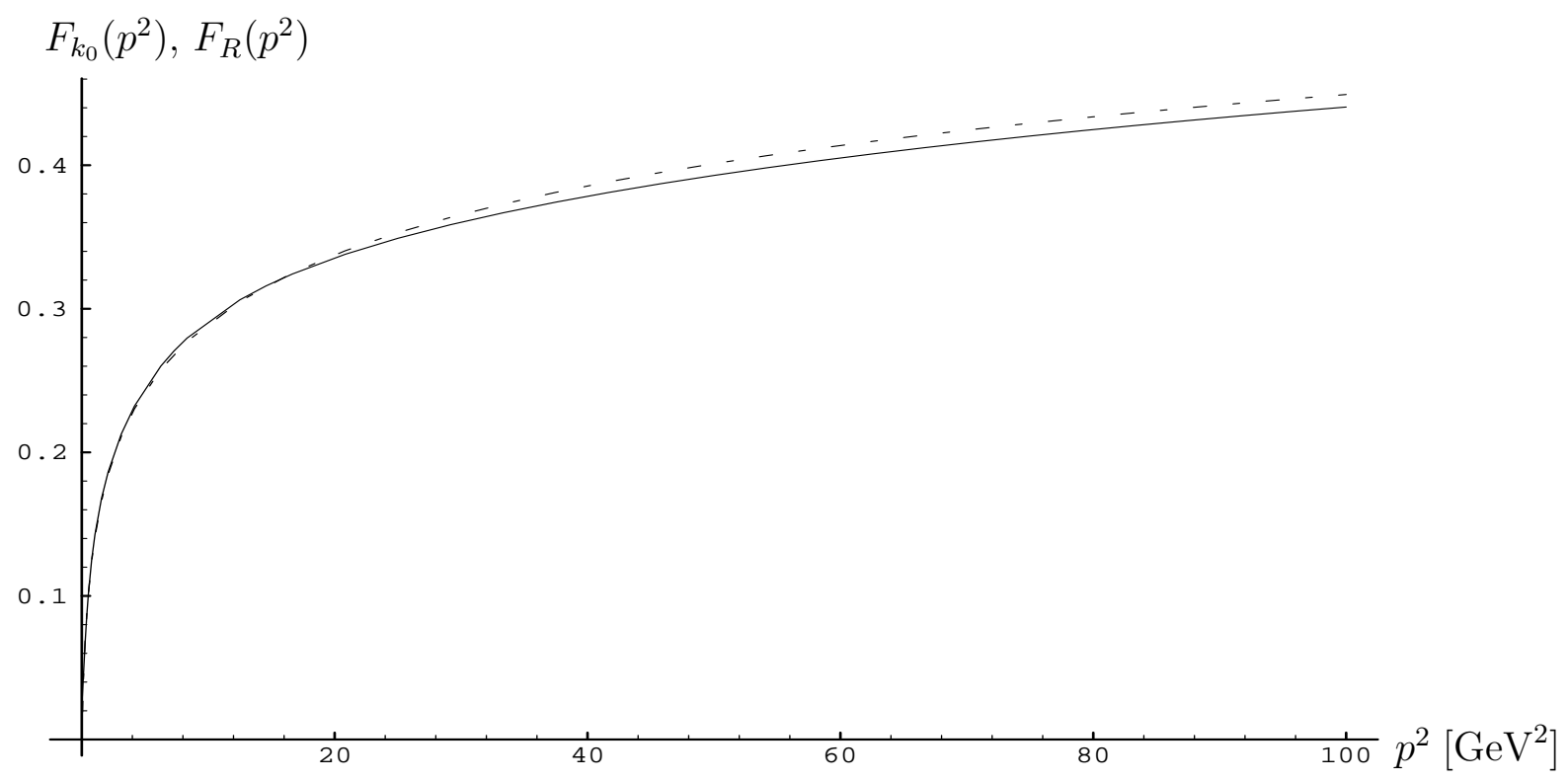

Figure 5

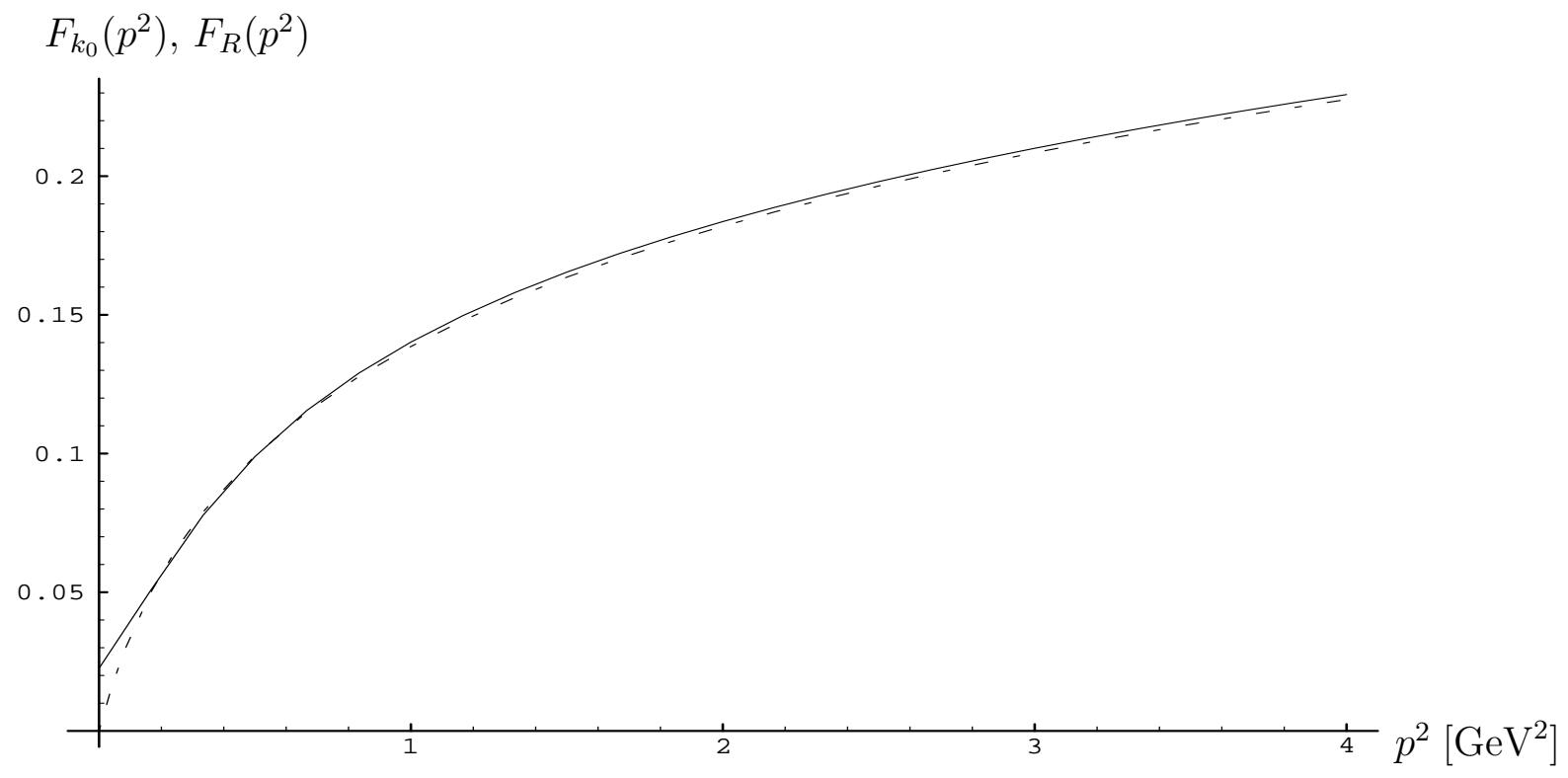

Figure 6 


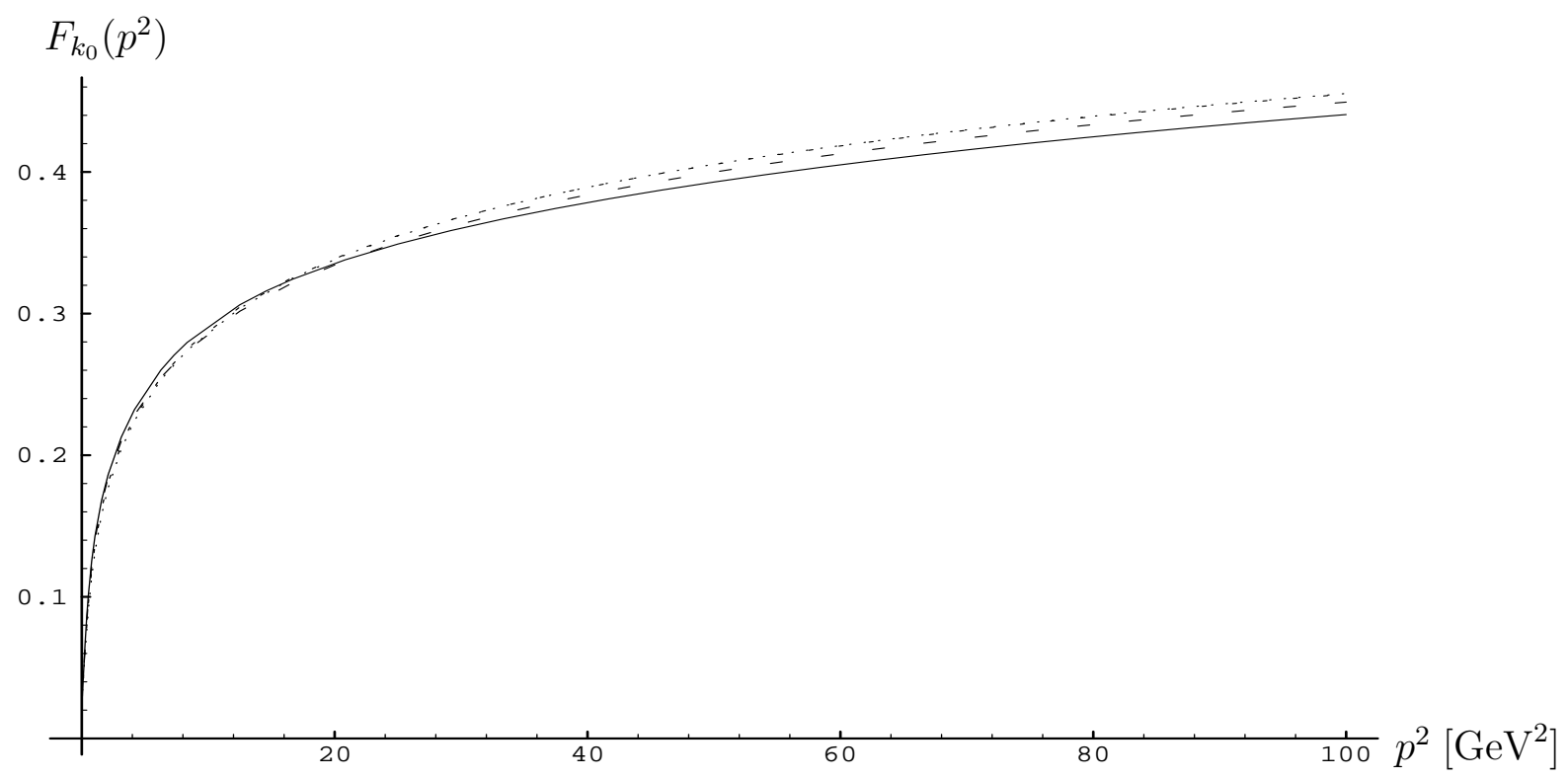

Figure 7

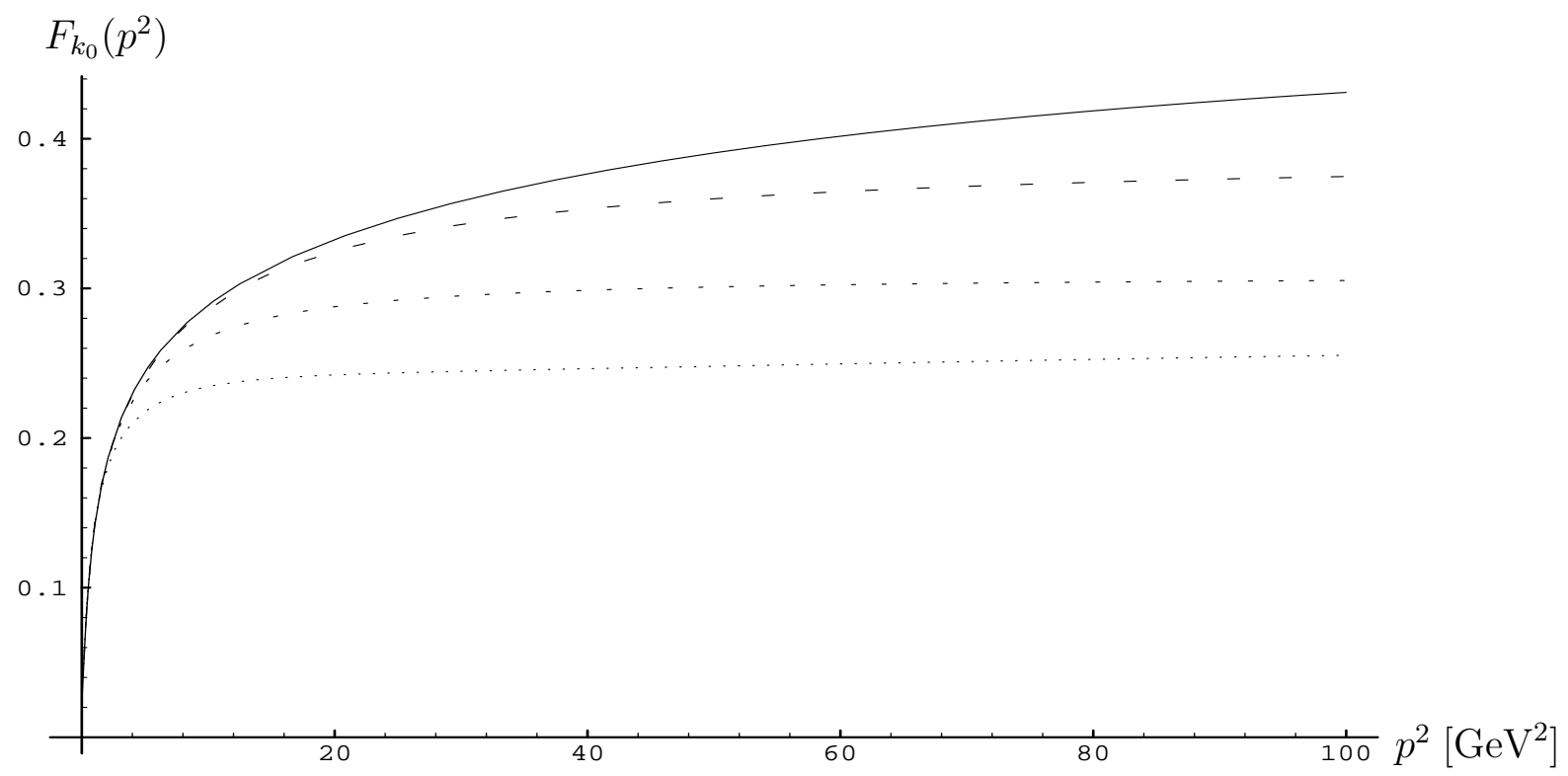

Figure 8 


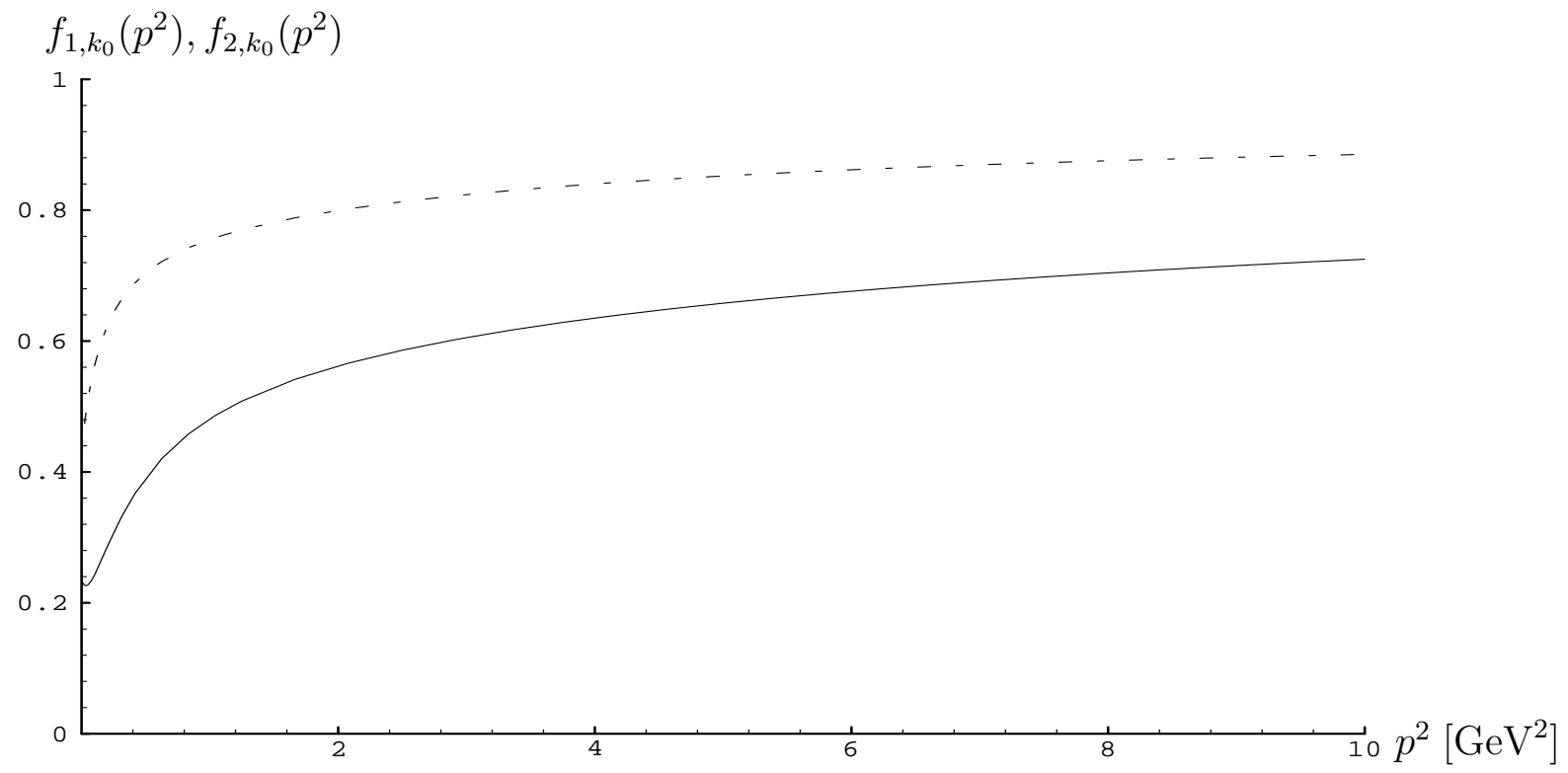

Figure 9 